\title{
Genetic diversity within the genus Francisella as revealed by comparative analyses of the genomes of two North American isolates from environmental sources
}

Shivakumara Siddaramappa', Jean F Challacombe ${ }^{1}$, Jeannine M Petersen ${ }^{2}$, Segaran Pillai ${ }^{3}$ and Cheryl R Kuske ${ }^{1^{*}}$

\begin{abstract}
Background: Francisella tularensis is an intracellular pathogen that causes tularemia in humans and the public health importance of this bacterium has been well documented in recent history. Francisella philomiragia, a distant relative of $F$. tularensis, is thought to constitute an environmental lineage along with Francisella novicida. Nevertheless, both F. philomiragia and F. novicida have been associated with human disease, primarily in immune-compromised individuals. To understand the genetic relationships and evolutionary contexts among different lineages within the genus Francisella, the genome of Francisella spp. strain TX07-7308 was sequenced and compared to the genomes of F. philomiragia strains ATCC 25017 and 25015, F. novicida strain U112, and F. tularensis strain Schu S4.
\end{abstract}

Results: The size of strain ATCC 25017 chromosome was 2,045,775 bp and contained 1,983 protein-coding genes. The size of strain TX07-7308 chromosome was 2,035,931 bp and contained 1,980 protein-coding genes. Pairwise BLAST comparisons indicated that strains TX07-7308 and ATCC 25017 contained 1,700 protein coding genes in common. NUCmer analyses revealed that the chromosomes of strains TX07-7308 and ATCC 25017 were mostly collinear except for a few gaps, translocations, and/or inversions. Using the genome sequence data and comparative analyses with other members of the genus Francisella (e.g., F. novicida strain U112 and F. tularensis strain Schu S4), several strain-specific genes were identified. Strains TX07-7308 and ATCC 25017 contained an operon with six open reading frames encoding proteins related to enzymes involved in thiamine biosynthesis that was absent in F. novicida strain U112 and F. tularensis strain Schu S4. Strain ATCC 25017 contained an operon putatively involved in lactose metabolism that was absent in strain TX07-7308, F. novicida strain U112, and F. tularensis strain Schu S4. In contrast, strain TX07-7308 contained an operon putatively involved in glucuronate metabolism that was absent in the genomes of strain ATCC 25017, F. novicida strain U112, and F. tularensis strain Schu S4. The polymorphic nature of polysaccharide biosynthesis/modification gene clusters among different Francisella strains was also evident from genome analyses.

Conclusions: From genome comparisons, it appeared that genes encoding novel functions have contributed to the metabolic enrichment of the environmental lineages within the genus Francisella. The inability to acquire new genes coupled with the loss of ancestral traits and the consequent reductive evolution may be a cause for, as well as an effect of, niche selection of $F$. tularensis. Sequencing and comparison of the genomes of more isolates are required to obtain further insights into the ecology and evolution of different species within the genus Francisella.

\footnotetext{
* Correspondence: kuske@lanl.gov

'Bioscience Division, Los Alamos National Laboratory, Los Alamos, NewMexico 87545, USA

Full list of author information is available at the end of the article
}

\section{Biomed Central}

(c) 2012 Siddaramappa et al.; licensee BioMed Central Ltd. This is an Open Access article distributed under the terms of the Creative Commons Attribution License (http://creativecommons.org/licenses/by/2.0), which permits unrestricted use, distribution, and reproduction in any medium, provided the original work is properly cited. 


\section{Background}

A small gram-negative bacterium that resembled members of the genus Pasteurella in many features was isolated in 1959 from a moribund muskrat found on the shoreline of a marsh on the Bear River Migratory Bird Refuge in northern Utah [1]. Based on morphological and biochemical characteristics, this bacterium was originally classified as Yersinia philomiragia (ATCC strain $25015^{\mathrm{T}}$ ). Additional isolations (ATCC strains 25016, 25017, and 25018) of bacteria similar to the muskrat pathogen were made in 1960 from surface water samples collected in marshy areas within few miles of the Bear River Research Station, Utah [1]. Subsequent studies indicated that ATCC strains $25015^{\mathrm{T}}, 25016,25017$, and 25018 were biochemically and genetically different from members of the genus Yersinia [2]. Between 1975 and 1987, fourteen isolates resembling Yersinia philomiragia were reported from clinical cases [3]. These human isolates and the 4 ATCC strains from Utah were reclassified based on biochemical characteristics, cellular fatty acid compositions, and DNA hybridization studies as Francisella philomiragia (philomiragia $=$ loving mirages, because of the mirages seen in the area where the first strains were found in Utah) in 1989 [4].

Most of the clinical isolates of $F$. philomiragia are from human cases associated with saltwater exposure and the first environmental isolates were from a marshy area that forms part of the Great Salt Lake waterway $[3,4]$. Not surprisingly, F. philomiragia is halotolerant and grows in media containing $6 \% \mathrm{NaCl}$, whereas Francisella tularensis does not [5]. Furthermore, in the laboratory, $F$. philomiragia is less fastidious than $F$. tularensis and does not require cysteine for growth [4]. F. philomiragia is considered an opportunistic pathogen and is most commonly isolated from patients with chronic granulomatous disease or those who experienced near-drowning in saltwater [3,4]. In contrast, $F$. tularensis is a highly infectious zoonotic agent and tularemia in healthy individuals exposed to contaminated freshwater sources has been reported [6]. Nevertheless, F. tularensis is generally not associated with saltwater exposure and is not known to cause near-drowningassociated pneumonia [7]. Isolations of F. philomiragia in the clinical laboratory have been sporadic and only four human cases have been reported in the last two decades [8-11]. A case of septicemia due to $F$. philomiragia has also been reported in a North Carolina dog that lived in a coastal town [12].

Recent attempts at direct isolation of Francisella spp. from environmental samples using cysteine heart agar with 9\% chocolatized sheep blood have yielded three strains (TX07-6608, TX07-7308, and TX07-7310) from seawater collected in the Galveston Bay area, Texas [13]. Comparisons of $16 \mathrm{~S}$ rRNA and $s d h A$ genes from these strains with those of other Francisella spp. (e.g., F. novicida strain U112 and F. tularensis strain Schu S4) have indicated that strain TX07-7308 is more similar to $F$. philomiragia than to $F$. tularensis [13]. A phylogenetic study based on $16 \mathrm{~S}$ rRNA genes of several members of Francisellaceae has clustered strain TX07-7308 with an uncultured bacterium clone SSW64Au obtained from the Salton Sea in California [14]. In addition, F. philomiragia has been detected in samples obtained from a brackish-water pond in Martha's Vineyard, Massachusetts [15]. Furthermore, a close taxonomic relationship between $F$. philomiragia and Francisella noatunensis, an emerging fish pathogen associated with marine environments, has also been established [16].

Francisella novicida is thought to constitute an environmental lineage along with $F$. philomiragia, and the genome of $F$. novicida strain U112 has been sequenced and compared to the genome sequences of $F$. tularensis strains pathogenic to humans [17]. The genomes of F. novicidalike strains Fx1 and 3523, which are clinical isolates from the USA and Australia, respectively, have also been sequenced and compared to the genome sequence of strain U112 [18]. In view of the ecological significance of $F$. philomiragia, the complete genome of strain ATCC 25017 (GenBank ID: 27853, a project of the DOE Joint Genome Institute) and a draft genome of strain ATCC $25015^{\mathrm{T}}$ (GenBank ID: 32411, a project of The Broad Institute) have been sequenced. Comparative genomic analyses of these strains with $F$. novicida and $F$. tularensis isolates have revealed major differences in metabolic competency and putative pathogenic characteristics $[19,20]$. In addition, a preliminary genomic sequence of $F$. noatunensis isolate GM2212 and its comparison to F. philomiragia have been reported [21]. The objectives of the present study were to sequence the genome of Francisella strain TX07-7308 and to identify the genetic differences between this strain and $F$. philomiragia strain ATCC 25017 using a comparative genomics approach. Since genetic relationships and evolutionary contexts could be better understood by whole-genome analyses of conserved operons, it was envisaged to include the genomes of different Francisella species and strains in the comparisons, when feasible.

\section{Methods}

Bacterial cultivation and chromosomal DNA extraction were performed at the Centers for Disease Control and Prevention, Fort Collins, using standard procedures $[13,22]$. Genomic library construction, sequencing, and finishing were performed at the Genome Science Facilities of Los Alamos National Laboratory as described previously [23-25]. Prediction of the number of subsystems and pairwise BLAST comparisons of protein sets within strains TX07-7308 and ATCC 25017 were performed using the Rapid Annotation using Subsystems 
Technology (RAST), which is a fully automated, prokaryotic genome annotation service [26]. Proteins deemed to be specific to each strain were compared against the NCBI non-redundant protein database to determine whether they were hypothetical or conserved hypothetical. If there was no adequate alignment with any protein (less than $25 \%$ identity or aligned region is less than $25 \%$ of the predicted protein length), the translated open reading frame (ORF) was named a hypothetical protein.

Multiple genome comparisons were performed using the 'progressive alignment' option available in the program MAUVE version 2.3.0. Default scoring and parameters were used for generating the alignment. A synteny plot was generated using the program NUCmer. The program uses exact matching, clustering, and alignment extension strategies to create a dot plot based on the number of identical alignments between two genomes. Prophage regions (PRs) were identified using Prophinder (http:// aclame.ulb.ac.be/Tools/Prophinder/), an algorithm that combines similarity searches, statistical detection of phage-gene enriched regions, and genomic context for prophage prediction. Insertion sequences (ISs) were identified by whole genome BLAST analysis of strains TX077308 and ATCC 25017 using the IS finder (http://www-is. biotoul.fr/). Gene acquisition and loss among the different strains were determined by comparing gene order, orientation of genes (forward/reverse), GC content of genes (\% above or below whole genome average), features of intergenic regions (e.g., remnants of IS elements, integration sites), and the similarity of proteins encoded by genes at a locus of interest ( $>90 \%$ identity at the predicted protein level for orthologs).

The Integrated Microbial Genomes System (http://img. jgi.doe.gov/cgi-bin/w/main.cgi) was used for comparing the genomes and identification of shared and unique protein encoding genes. The phylogenetic profiling tool within IMG was used to find single gene homologs. Three 3-way comparisons were performed using different genome orders, and the lowest number was used to represent the core genes in the venn diagram. Pairwise comparisons were performed bidirectionally for each pair of genomes and the lowest number was used in the diagram. Strain-specific genes were obtained by subtracting the larger number of common genes from the total number of genes in the genome. The specific parameters used for the analysis were: Evalue: 1e-05; minimum percent identity: $30 \%$; algorithm: by present/absent homologs; minimum taxon percent with homologs: $100 \%$.

Multiple sequence alignments for phylogenetic analyses of strains TX07-7308 and ATCC 25017 were performed using the program MUSCLE available at the website http://www.phylogeny.fr/[27,28]. The alignment was followed by a bootstrapped $(n=100)$ neighbor joining method for inferring the phylogenies [29]. Only fulllength $16 \mathrm{~S}$ rRNA and succinate dehydrogenase $(s d h A)$ gene sequences from high quality, finished Francisella genomes were included in these comparisons.

\section{Results and discussion}

\section{General genome comparisons}

The chromosome of strain ATCC 25017 was 9,844 bp larger than that of strain TX07-7308. Although the chromosomes of strains ATCC 25017, ATCC 25015, and TX077308 had minor differences in size, their average GC content and the percentage of sequence that encodes proteins were similar (Table 1). Whole genome alignment using MAUVE showed the presence of extensive blocks of homologous regions among the chromosomes of strains ATCC 25017 and TX07-7308 (Figure 1). NUCmer analyses

Table 1 Comparison of the genomes of five different strains within the genus Francisella

\begin{tabular}{|c|c|c|c|c|c|}
\hline Genome feature & $\begin{array}{l}\text { F. philomiragia } \\
\text { ATCC } 25017\end{array}$ & $\begin{array}{l}\text { Francisella spp. } \\
\text { TX07-7308 }\end{array}$ & $\begin{array}{l}\text { F. philomiragia } \\
\text { ATCC } 25015^{\top}\end{array}$ & $\begin{array}{l}\text { F. novicida } \\
\text { U112 }\end{array}$ & $\begin{array}{l}\text { F. tularensis } \\
\text { WY96-3418 }\end{array}$ \\
\hline Chromosome size (bp) & 2045775 & 2035931 & 1985978 & 1910031 & 1898476 \\
\hline Number of protein-coding genes & 1915 & 1976 & 1892 & 1733 & 1634 \\
\hline Overall coding density (\%) & 91 & 91 & 91 & 89 & 79 \\
\hline Number of subsystems ${ }^{1}$ & 306 & 302 & 308 & 253 & 250 \\
\hline GC content (\%) & 32.57 & 32.9 & 32.52 & 32.48 & 32.27 \\
\hline $5 S$ rRNA genes ${ }^{2}$ & $3+1$ & $3+1$ & $3+1$ & $3+1$ & $3+1$ \\
\hline 235 rRNA genes ${ }^{2}$ & 3 & 3 & 3 & 3 & 3 \\
\hline $16 S$ rRNA genes ${ }^{2}$ & 3 & 3 & 3 & 3 & 3 \\
\hline tRNA genes & 39 & 39 & 35 & 38 & 38 \\
\hline GenBank accession number & CP000937 & CP002872 & ABYY00000000 & CP000439 & СР000608 \\
\hline
\end{tabular}

${ }^{1}$ Type strain, draft genome.

1 Subsystems predicted by RAST server.

2 Each strain contains three rRNA operons (5S-23S-16S) and an orphan 5S rRNA fragment. 


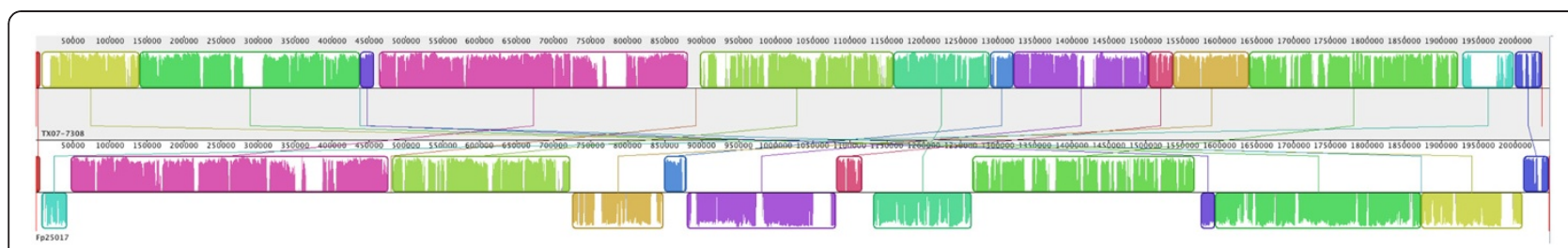

Figure 1 Alignment of the chromosomes of strains TX07-7308 (top) and ATCC 25017 (bottom) using MAUVE 2. Identically colored boxes, known as locally collinear blocks (LCBs), depict homologous regions in the two chromosomes. The edges of LCBs indicate chromosome rearrangements due to recombination, insertions, and/or inversions. Sequences of strain ATCC 25017 inverted in relation to those of strain TX07-7308 are shown as blocks below the horizontal line. The vertical lines connecting the LCBs point to regions of homology among the two chromosomes. Numbers above the maps indicate nucleotide positions within the respective chromosomes.

revealed that the chromosome of strain ATCC $25017 \mathrm{had}$ many gaps, translocations, and/or inversions when compared to that of strain TX07-7308 (Figure 2). Detailed sequence examination indicated that some of these gaps and/ or inversions were associated with integrative elements. Strain TX07-7308 contained a genomic island (920,244 bp to $927,148 \mathrm{bp}, 29.89 \% \mathrm{GC}$ ) that was absent in strain ATCC 25017, but was present in F. novicida strain U112 (369,322 bp to 376,086 bp, $30.7 \%$ GC). Strain TX07-7308 contained yet another genomic island $(1,940,355$ bp to $1,971,897 \mathrm{bp}$, $28.98 \%$ GC) that was also absent in strain ATCC 25017, but was partially present in F. novicida strain U112 (e.g., 40,574 bp to $61,538 \mathrm{bp}, 28.8 \%$ GC). Strain ATCC 25017 contained a genomic island $(1,981,253 \mathrm{bp}$ to $1,996,171 \mathrm{bp}$, $30 \%$ GC) that was absent in strain TX07-7308 and F. novicida strain U112. Furthermore, strain TX07-7308 lacked plasmids, but strain ATCC 25017 contained a single plasmid [30].

A three-way comparison of the chromosomes of strains U112, ATCC 25017, and TX07-7308 revealed that they encoded 1,468 orthologous protein-coding genes (bidirectional best hits). Strain ATCC 25017 chromosome encoded 196 protein-coding genes with no homologs in strains TX07-7308 and U112. Strain TX077308 chromosome encoded 249 protein-coding genes with no homologs in strains U112 and ATCC 25017.

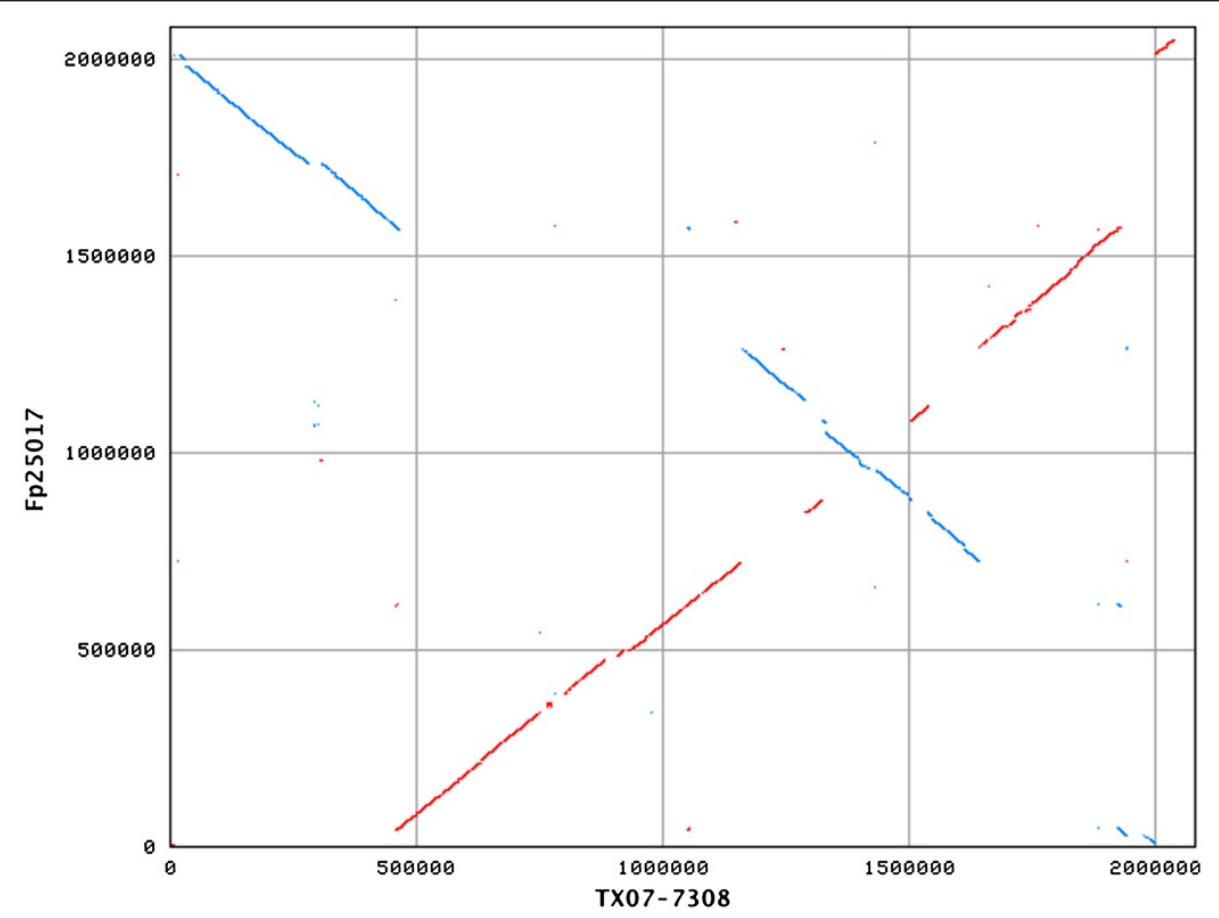

Figure 2 Synteny plot of the chromosomes of strains TX07-7308 and ATCC 25017 generated by NUCmer. This plot shows regions of identity between the two chromosomes based on pair-wise alignments. Strain TX07-7308 sequence is represented on the X-axis. Strain ATCC 25017 sequence is represented on the Y-axis. Plus strand matches are slanted from the bottom left to the upper right corner and are shown in red. Minus strand matches are slanted from the upper left to the lower right and are shown in blue. The number of dots/lines shown in the plot is the same as the number of exact matches found by NUCmer. Numbers indicate nucleotide positions within the respective chromosomes. 
Strain U112 encoded 211 protein-coding genes with no homologs in strains TX07-7308 and ATCC 25017 (Figure 3). In strain ATCC 25017, 507 predicted genes could not be assigned a function based on BLAST analysis and have therefore been annotated as encoding hypothetical or conserved hypothetical proteins. In strain TX07-7308, 447 predicted genes have been annotated as encoding hypothetical or conserved hypothetical proteins.

\section{Ribosomal RNA operons and tRNA genes}

Bacterial rRNA operons generally contain the $16 \mathrm{~S}, 23 \mathrm{~S}$, and 5S rRNA genes linked together by one or more tRNA genes located in the internal transcribed spacer [31]. Chromosomes of most members of the genus Francisella sequenced to date, including strains ATCC 25017 and TX07-7308, contained three copies of the $16 \mathrm{~S}$ rRNA-tRNA ${ }^{\text {Ile }}$-tRNA ${ }^{\text {Ala }}$-23S rRNA-5S rRNA operon. Although the three rRNA operon sequences were remarkably homogenous within any genome, some degree of heterogeneity was obvious between the F. philomiragia and F. tularensis/novicida genomes (data not shown). Notably, the chromosomes of strains ATCC 25017 and TX07-7308 contained an unlinked 119 bp 5S rRNA sequence $(380,981 \mathrm{bp}$ to $381,099 \mathrm{bp}$ and $1,883,624 \mathrm{bp}$ to $1,883,742 \mathrm{bp}$, respectively) that was also present in other members of the genus Francisella (e.g., FTN_0501 in F.

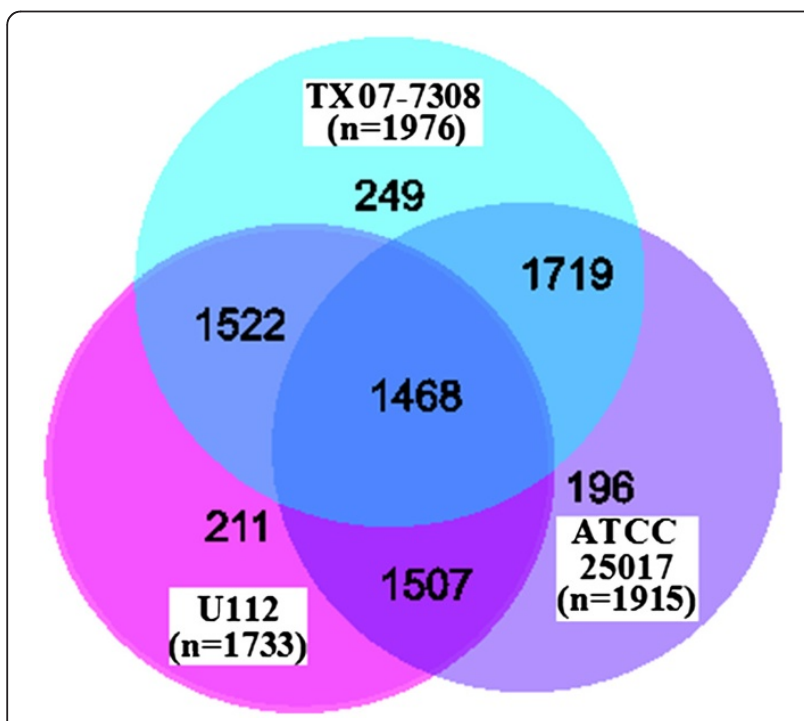

Figure 3 Venn diagram depicting the shared (orthologs) and unique protein coding genes in the genomes of Francisella spp. strain TX07-7308, F. philomiragia strains ATCC 25017, and F. novicida strain U112. Numbers in parenthesis indicate the total number of protein coding genes in the genomes of each of the three strains. Strains ATCC 25017 and TX07-7308 had 1719 orthologous protein coding genes, strains ATCC 25017 and U112 had 1507 orthologous protein coding genes, whereas strains TX07-7308 and U112 had 1522 orthologous protein coding genes. novicida strain U112 and FTT_r01 in F. tularensis strain Schu S4). Furthermore, the positions of the three rRNA operons were not conserved in the chromosomes of strains ATCC 25017 and TX07-7308, but they were relatively conserved among the chromosomes of F. novicida and F. tularensis strains.

A positive correlation between the abundance of tRNA and rRNA genes among bacterial genomes has been observed [32]. Typically, bacteria with three 16S rRNA genes are expected to contain $\sim 40$ tRNA genes [31]. Not surprisingly, members of the genus Francisella contain $37-39$ tRNA genes ( $1.8 \%$ of the genome), representing all 20 amino acids. Although some redundancy of tRNA genes was apparent among all Francisella genomes (e.g., four copies of tRNA $\left.{ }^{\mathrm{Leu}}\right)$, they each contained a single copy of tRNA ${ }^{\text {Asn }}, \mathrm{tRNA}^{\mathrm{Asp}}, \mathrm{tRNA}^{\text {Cys }}, \mathrm{tRNA}^{\mathrm{Gln}}, \mathrm{tRNA}^{\text {Glu }}$, tRNA $^{\text {His }}$, tRNA ${ }^{\text {Phe }}$, tRNA ${ }^{\text {Pro }}$, tRNA ${ }^{\text {Trp }}$, and tRNA $^{\text {Tyr }}$ genes. In all of the publicly available Francisella genomes, genes encoding tRNA ${ }^{\text {Ile }}$ and $\mathrm{tRNA}^{\text {Ala }}$ were never present outside of the rRNA operons. This is generally true of most other bacteria and the occurrence of genes encoding tRNA $^{\text {Ile }}$ and tRNA $^{\text {Ala }}$ genes within the rRNA operons has also been noted in Escherichia coli $\mathrm{K} 12$ and Rhodopseudomonas palustris [33,34]. Furthermore, almost all Francisella genomes contained genes encoding putative aminoacyl tRNA synthetases specific for each of the 20 amino acids. They also contained a single gene encoding a putative peptidyl-tRNA hydrolase (e.g., Fphi_1584, FTN_1003, and FTT_0680c).

\section{Integrative and transposable elements}

Short sequence repeats, which include insertion sequences (IS), are the hallmark of F. tularensis genomes and these IS elements are thought to be generally stable among different isolates despite their diverse geographical origins $[17,35]$. Pair-wise BLASTN analyses indicated that IS elements were less abundant in the genome of strain TX07-7308 in comparison to those of F. tularensis strains Schu S4 and OSU18, which contained at least six categories of IS elements (ISFtu1ISFtu6). Whole genome BLASTP analyses showed that strains ATCC 25017 and TX07-7308 each contained a single copy of the ISFtu2 sequence (Fphi_0257, 247 aa; F7308_1638, 235 aa; 91\% identity), but they lacked ISFtu1 and ISFtu3-ISFtu6 sequences (Additional file 1: Table S1). Furthermore, strains TX07-7308 and ATCC 25017 contained at least 4 truncated loci related to IS1016 (F7308_1212, F7308_1886, F7308_1888, and Fphi_1491; Additional file 1: Table S1).

In strain TX07-7308, the locus adjacent to F7308 1638 encoded an integral membrane protein (F7308_1634, 343 aa), a phosphoserine phosphatase (F7308_1635, 216 aa), a lignostilbene-alpha, beta-dioxygenase (F7308_ 1636, 99 aa), and a beta carotene dioxygenase (F7308_ 
1637, 344 aa). Interestingly, the homologs of these ORFs in strain ATCC 25017 (Fphi_0135-0138) were associated with Fphi_0139, which encoded a putative integrase (314 aa). Strain ATCC 25017 contained 41 full-length and 5 truncated orthologs of Fphi_0139 (Additional file 1: Table S1). Whereas an ortholog of Fphi_0139 was present in Francisella piscicida strain GM2212 (GenBank locus EU492905, protein_id ACA58079), it was absent in all other publicly available Francisella genomes, including strain ATCC 25015.

Strains ATCC 25017 and TX07-7308 also contained a putative mutator family transposase (Fphi_0511, 410 aa; F7308_0014, 211 aa; 85\% identity, Additional file 1: Table $\bar{S} 1$ ) that was absent in all other publicly available Francisella genomes, except strain ATCC 25015. In strain ATCC 25017, this transposable element was associated with a gene encoding a putative chitinase (Fphi_0512, 617 aa) whose ortholog was present in strain ATCC 25015, but not in strain TX07-7308 and all other publicly available Francisella genomes. Strain TX077308 mutator family transposase was associated with genes encoding a putative chitinase (F7308_0017, 942 aa) and a two-component response regulator (F7308_0016, 239 aa) whose orthologs were present in strain ATCC 25015, but not in strain ATCC 25017 and all other publicly available Francisella genomes. Furthermore, strain ATCC 25017 contained a locus (Additional file 1: Table S1) encoding a putative Tn1546 family transposase (Fphi_1740, 552 aa), an integrase (Fphi_1741, ortholog of Fphi_0139, 314 aa), a Tn3 family transposase (Fphi_1742, 238 aa), a transposase-like protein (Fphi_1743, 137 aa), and an invertase/recombinase/resolvase-like protein (Fphi_1744, 189 aa). These genes were absent in all other publicly available complete Francisella genomes, including strain TX07-7308. Several genes encoding putative phage integrases were also identified in the genomes of strains ATCC 25017 and TX07-7308 (Additional file 1: Table S1).

\section{Type IV pili, oligopeptide ABC transporter system, Francisella pathogenicity island, and iron metabolism genes}

Several species of pathogenic gram-negative bacteria contain type IV pili, also known as fimbriae, which facilitate bacterial adhesion to host cells, biofilm formation, and twitching motility [36]. Structures resembling type IV pili have been observed on the surface of $F$. tularensis live vaccine strain (LVS) and F. novicida strain U112 using transmission electron microscopy $[37,38]$. Recent evidence suggests that type IV pili are required for virulence of F. tularensis and comparisons of available Francisella genomes have revealed the heterogeneity of genes putatively involved in the assembly of type IV pili $[39,40]$. Strains ATCC 25017 and TX07-7308 contained several ORFs that encoded putative proteins related to the assembly of type IV pili (Table 2). Although most of these genes were found in different locations on the two chromosomes, two gene clusters were conspicuous from gross comparisons (Fphi_0006-0010/F7308_12361232 and Fphi_0422-0424/F7308_0429-0427). Since many of the genes putatively involved in the biosynthesis of type IV pili in strains ATCC 25017 and TX077308 were also present in F. novicida and F. tularensis strains (data not shown), the biochemical and functional similarity of type IV pili among these bacteria cannot be ruled out. Nevertheless, the role of type IV pili in the survival of $F$. philomiragia in the environment needs to be carefully studied, especially in light of recent reports of the ability of this bacterium to form biofilms and interact with the aquatic protist Acanthamoeba castellanii [41].

Several bacterial species possess oligopeptide permeases (Opp), which are ATP-binding cassette transporters of oligopeptides [42]. The imported oligopeptides serve as a source of nitrogen for the cell and may also have a role in signal transduction and pathogenesis [43]. A typical bacterial oligopeptide transport system consists of five proteins encoded by the oppABCDF operon [42,43]. It has been reported that $o p p D$ and $o p p F$ occur as separate ORFs in Type A strains of F. tularensis, but a 960 bp internal deletion has caused the disruption of $o p p D$ and $o p p F$ coding sequences in Type B strains of F. tularensis [19]. Genome comparisons indicated that F. philomiragia strain ATCC 25017 and F. novicida strain U112 contained an opp locus (Fphi_1024-1029 and FTN_1589-1593, respectively). Within this locus, FTN_1590 encoded a putative fulllength OppD protein (322 aa), but Fphi_1027 was annotated as a pseudogene because of a frame-shift mutation (Table 2). Although the opp locus was present in a complete or truncated form in most currently available Francisella genomes, it was absent in strain TX07-7308. It is possible that strain TX07-7308 lost the ancestral opp locus during reductive evolution or it represents a lineage that evolved before the acquisition of the opp locus by the F. philomiragia clade.

A cluster of 17-19 genes has been proposed to constitute the Francisella pathogenicity island (FPI) and is found in a single copy in F. novicida strain U112 (FTN_1309-1325), but is duplicated in the genomes of $F$. tularensis $[17,44]$. Genome comparisons revealed that strains ATCC 25017 and TX07-7308 also contained a cluster of genes related to the FPI (Fphi_1363-1367/ F7308_1001-1005 and Fphi_1369-1377/F7308_10071016). The predicted proteins within the putative FPIs of strains ATCC 25017 and TX07-7308 had an average identity of $84.5 \%$ (the identity range was $59-96 \%$ ). The order and orientation of genes within the FPI of strains ATCC 25017 and TX07-7308 were similar. Furthermore, 
Table 2 Identification of pilus biosynthesis, oligopeptide transport, glucuronate metabolism, and lactose metabolism genes

\begin{tabular}{|c|c|c|}
\hline $\begin{array}{l}\text { Function } \\
\text { Locus tag (Protein) }\end{array}$ & Annotation & $\begin{array}{l}\text { Closest homolog outside Francisella } \\
\text { locus tag, protein, identity, E-value }\end{array}$ \\
\hline \multicolumn{3}{|l|}{ Pilus biosynthesis $^{1}$} \\
\hline Fphi_0006 (582 aa) & Type IV pili secretin component (PilQ) & Csal_0612, 668 aa, 36\%, 7e-84 \\
\hline Fphi_0007 (196 aа) & Type IV pili lipoprotein (PilP) & Rmet_3269, 179 aa, 37\%, 2e-05 \\
\hline Fphi_0008 (199 aа) & Type IV pili glycosylation protein (PilO) & Lferr_0885, 217 aa, 24\%, 2e-04 \\
\hline Fphi_0009 (187 aа) & Type IV pili associated protein (PilN) & Lferr_0888, 197 aa, 26\%, 5e-05 \\
\hline Fphi_0010 (334 aa) & Type IV pili, pilus assembly protein & None \\
\hline Fphi_0117 (592 aa) & Type IV pili ATPase (PilB/PulE) & Shewmr4_0420, 569 aa, 48\%, 4e-147 \\
\hline Fphi_0118 (410 aa) & Type IV pili polytopic inner membrane protein (PilC/PulF) & Tgr7_0786, 403 aа, 42\%, 7e-85 \\
\hline Fphi_0157 (194 aa) & Type IV pili, pilus assembly protein (FimT) & Shal_1142, 171 aa, 30\%, 6e-11 \\
\hline Fphi_0422 (152 aa) & Type IV pili fiber building block protein (PilA/PilE) & Noc_2540, 147 aa, 35\%, 7e-15 \\
\hline Fphi_0423 (145 aa) & Type IV pili, pilus assembly protein (PilA/PilE) & CV_4209, 155 aa, 33\%, 5e-12 \\
\hline Fphi_0424 (409 aa) & Type IV pili, pilus assembly protein (PilA/PilE) & NMB0018, 170 aa, 30\%, 3e-09 \\
\hline Fphi_0449 (313 аa) & Type IV pili, pilus assembly protein (PilA/PilE) & CtCNB1_4760, 144 aa, 28\%, 3e-05 \\
\hline Fphi_0522 (304 aa) & Type IV pilus assembly protein PilW & None \\
\hline Fphi_0763 (149 aa) & Type IV pili, pilus assembly protein & Tola_2518, 128 aa, 36\%, 2e-15 \\
\hline Fphi_0996 (342 aа) & Twitching motility protein (PilT) & SO_3351, 345 aa, 58\%, 4e-106 \\
\hline Fphi_1136 (337 aa) & Type IV pilus assembly protein (PilW) & None \\
\hline Fphi_1587 (282 aa) & Type IV pili leader peptidase and methylase (PilD) & Maqu_2683, 291 aa, 41\%, 9e-55 \\
\hline Fphi_1689 (297 aa) & Type IV pilus biogenesis/stability protein (PilW) & Kkor_1840, 263 aa, 30\%, 1e-09 \\
\hline Fphi_1748 (111 aа) & Type IV pilin (PilA) & Hsero_0660, 168 aa, 28\%, 3e-06 \\
\hline \multicolumn{3}{|c|}{ Oligopeptide transport ${ }^{2}$} \\
\hline Fphi_1024 (558 aа) & ABC-type oligopeptide transport system, periplasmic component (OppA) & Rahaq_2640, 546 aa, 38\%, 1e-90 \\
\hline Fphi_1025 (312 aa) & Oligopeptide $A B C$ transporter inner membrane protein (OppB) & EAMY_1936, 306 aa, 47\%, 3e-77 \\
\hline Fphi_1026 (286 aa) & Oligopeptide transport system permease protein (OppC) & VP2089, 300 aa, 53\%, 2e-84 \\
\hline Fphi_1027 (219 aa) & Oligopeptide transport system permease protein (OppD) & CV_4326, 333 aa, 61\%, 3e-137 \\
\hline Fphi_1029 (324 aa) & Oligopeptide transport ATP-binding protein (OppF) & NT05HA_1387, 332 aа, 58\%, 5e-105 \\
\hline \multicolumn{3}{|c|}{ Glucuronate metabolism $^{3}$} \\
\hline F7308_1388 (520 aa) & Rhamnogalacturonide transporter (RhiT) & KPK_1307, 502 aa, 44\%, 2e-116 \\
\hline F7308_1389 (776 aa) & Alpha-glucosidase & BL00280, 802 aa, 50\%, 0.0 \\
\hline F7308_1390 (490 aa) & D-mannonate oxidoreductase (UxuB) & CJA_0180, 492 aa, 42\%, 3e-105 \\
\hline F7308_1391 (396 aa) & Mannonate dehydratase (UxuA) & PROSTU_04181, 396 aa, 59\%, 5e-135 \\
\hline F7308_1392 (321 aa) & 2-keto-3-deoxygluconate kinase (KdgK) & Sde_1269, 296 aa, 43\%, 9e-59 \\
\hline F7308_1393 (182 aa) & KDPG Aldolase (KdgA) & BC1003_2949, 213 aa, 37\%, 4e-34 \\
\hline F7308_1394 (471 aa) & Glucuronate isomerase (UxaC) & Sde_1272, 471 aa, 51\%, 3e-140 \\
\hline F7308_1395 (463 aа) & D-xylose-proton symporter (XyIT) & CBUD_1731, 463 aа, 43\%, 3e-91 \\
\hline F7308_1396 (325 aa) & Inositol oxygenase & 56727 Miox, 285 aa, 37\%, 3e-47 \\
\hline \multicolumn{3}{|l|}{ Lactose metabolism ${ }^{4}$} \\
\hline Fphi_0309 (655 aa) & Beta-galactosidase & BMD_1886, 651 aa, 42\%, 2e-159 \\
\hline Fphi_0310 (394 aа) & Sugar transport protein & ZP_07631419, 394 aa, 31\%, 2e-44 \\
\hline
\end{tabular}

${ }^{1}$ Homologs of these genes are also found in strains TX07-7308 and ATCC 25015.

${ }^{2}$ Homologs of these genes are also found in strain ATCC 25015, but absent in strain TX07-7308.

${ }^{3}$ Homologs of these genes are also found in strain ATCC 25015, but absent in strain ATCC 25017.

${ }^{4}$ Homologs of these genes are also found in strain ATCC 25015, but absent in strain TX07-7308. 
the homolog of FTN_1314, which encodes a hypothetical protein in F. novicida strain U112, was truncated in strain TX07-7308 (F7308_1011) and homologs of FTN_1318-FTN_1320 (encoding a hypothetical protein, a pathogenicity determinant protein, and a hypothetical protein, respectively) were absent in strains ATCC 25017 and TX07-7308.

The biosynthesis of a polycarboxylate siderophore in F. tularensis strain Schu S4 and F. novicida strain U112 has been described previously $[45,46]$. Strains ATCC 25017 and TX07-7308 contained the ferric uptake regulator gene (fur, Fphi_0928/F7308_0528) and a cluster of genes putatively involved in the biosynthesis of a polycarboxylate siderophore (Fphi_0922-0926 and F7308_ 0522-0526). An ortholog of strain Schu S4 fup $\bar{A}$ (FTT_0918), whose product is required for efficient utilization of siderophore-bound iron [47], was also found in strains ATCC 25017 and TX07-7308 (Fphi_ 0393/F7308_0459). The presence of FPI-related genes, fur, and fupA in almost all Francisella genomes suggests that these functions are essential for their survival in the environment and/or host.

\section{Genetics of uronic acid metabolism}

Some bacteria have evolved mechanisms for the metabolism of uronic acids and uronates using the Entner-Doudoroff pathway [48]. In this pathway, $\alpha$-D-glucuronic acid (GlcUA) is converted into 2-keto-3-deoxygluconate (KDG) by a threestep process. The subsequent phosphorylation of KDG yields 2-keto-3-deoxy-6-phosphogluconate (KDPG), which is finally cleaved to produce glyceraldehyde 3-phosphate (G3P) and pyruvate. Comparative genomic analyses indicated that strains TX07-7308 and ATCC 25015 contained a cluster of nine ORFs that appeared to constitute a polycistronic operon (F7308_1388-1396). These ORFs encoded putative proteins related to enzymes involved in GlcUA catabolism (Table 2). Similar operons were also identified in F. novicida-like strains Fx1 and 3523 [18].

The predicted mannonate dehydratase, 2-keto-3deoxygluconokinase, KDPG aldolase, and glucuronate isomerase proteins from strain TX07-7308 had 57\%, $37 \%, 36 \%$, and $28 \%$ identities to E. coli UxuA (ECs5281), KdgK (ECs4406), KdgA (ECs2560), and UxaC (ECs3974), respectively (E-values $=8 \mathrm{e}-136$ to $3 \mathrm{e}-29)$. These enzymes catalyze the dehydration of D-mannonate to KDG, phosphorylation of KDG, cleavage of KDPG to pyruvate and G3P, and the conversion of D-glucuronate to D-fructuronate, respectively [48]. The GlcUA utilization gene cluster of strain TX07-7308 had some similarities to that of Bacillus stearothermophilus T-6, which has been predicted to metabolize GlcUA akin to E. coli and Bacillus sutbtilis [49].

The ORFs encoding a putative inositol oxygenase in strains TX07-7308 and ATCC 25015 had no bacterial homologs in the public databases outside of the genus Francisella. However, strain TX07-7308 inositol oxygenase had 37\% identity to Mus musculus myo-inositol oxygenase (56727 Miox, E-value = 5e-54), which catalyzes the conversion of myo-inositol to GlcUA [50]. Myo-inositol and its derivatives are ubiquitous among eukaryotes and archaea, but their synthesis and metabolism is believed to be less common among bacteria [51]. Although none of the F. tularensis genomes sequenced to date had ORFs encoding proteins putatively involved in the transport and/or metabolism of myo-inositol, most of them had a suhB homolog (e.g., FTT_1382 in strain Schu S4). A $\operatorname{suh} B$ homolog was also found in the genomes of strains TX07-7308 and ATCC 25017 (F7308_0980 and Fphi_1342). This evolutionarily conserved gene encoded inositol-1-monophosphatase, which hydrolyzes myoinositol-1-phosphate to yield free myo-inositol [52,53]. Thus, it appears that most members of Francisella can convert myo-inositol-1-phosphate to free myo-inositol. However, only some strains may be able to utilize myoinositol to synthesize GlcUA, which is then metabolized using the Entner-Doudoroff pathway.

\section{Atypical lac operons}

The lac operon, which contains the structural genes encoding proteins that facilitate lactose metabolism, is found in a variety of bacteria. Lactose is imported into the cell as a free sugar by means of a permease and the enzyme $\beta$-galactosidase hydrolyzes this disaccharide into galactose and glucose [54]. Genome comparisons revealed that strains ATCC 25015 and ATCC 25017, but not strain TX07-7308, contained a cluster of two ORFs that appeared to constitute an operon (Fphi_0309-0310, Table 2). Similar lac operons were also identified in $F$. novicida-like strains Fx1 and 3523 [18]. Strain ATCC 25017 predicted LacZ had $29 \%$ identity to the $\beta$ galactosidase (AAF16519, BgaB, E-value $=2 \mathrm{e}-79$ ) of Carnobacterium maltaromaticum [55] and 26\% identity to the $\beta$-galactosidase (O07012.2, GanA, E-value $=8 \mathrm{e}-76)$ of Bacillus subtilis [56], but was unrelated to the $\beta$ galactosidase of E. coli. Strain ATCC 25017 LacY had $25 \%$ identity to the putative oligogalacturonide transporter (NP_752266, E-value $=3 \mathrm{e}-07$ ) of E. coli CFT073 and $22 \%$ identity to the putative sugar transporter (YP_081973, E-value = 1e-08) of Bacillus cereus. However, genes encoding the galactoside $\mathrm{O}$-acetyltransferase $(\operatorname{lac} A)$ and the regulatory protein $(l a c I)$ were not found in strains ATCC 25015 and ATCC 25017. In strain ATCC 25017, an ORF (Fphi_0306) encoding a putative transposase/integrase-like protein was found near the lac operon. Furthermore, several F. tularensis genomes (e.g., strains Schu S4, WY96-3418, OSU18, and FSC147) contained a truncated/vestigial lac $Z$, but lacked a $l a c Y$ (data not shown). 
Although the classic lac operon of $E$. coli consists of three ORFs (lacZYA), which are regulated by the product of the adjacent lacI repressor gene, bacteria containing only lacZY or lac $Z$ have been identified [57-59]. While the evolutionary origin of the E. coli lac operon is uncertain [59], the occurrence of lac genes near integrative and conjugative elements and the identification of $E$. coli-like lac operons in some gram-positive bacteria suggest their lateral mobility [60-62]. From genome comparisons, it appeared that the last common lactose-utilizing ancestor of F. philomiragia strain ATCC 25017 and F. tularensis strains may have acquired the lac $Z Y$ operon by transposon-mediated horizontal transfer. Nevertheless, detailed phylogenetic analyses are required to establish the evolutionary origin of the Francisella lac operon. The loss of the lacZY operon in most F. tularensis strains may be due to niche selection or through genetic drift, and a similar mechanism for some members of Enterobacteriaceae has been proposed [59]. The ability to metabolize lactose probably affords strains ATCC 25015 and ATCC 25017 a growth advantage in environments where the sugar is present and these may represent a subset of Francisella that have retained an ancestral copy of the lac operon. The lacZ gene identified in this report could be useful in studies involving pathogenic F. tularensis strains that require a native reporter protein.

\section{Genetics of thiamine, riboflavin, folate, biotin, and siroheme biosynthesis}

Thiamine pyrophosphate (Vitamin $B_{1}$ ) is involved in several microbial metabolic functions [63]. Prokaryotes have evolved elaborate mechanisms to either synthesize this important co-factor de novo or acquire it from their niche [64]. Thiamine biosynthesis (TBS) in most bacteria is accomplished by two major pathways; one involves the formation of hydroxymethylpyrimidine pyrophospate (HMP-PP) from aminoimidazole ribotide using ThiC and ThiD and the other involves the formation of hydroxyethylthiazole phosphate (HET-P) using ThiS, ThiF, ThiG, and ThiO. The enzyme thiamine phosphate synthase (ThiE) combines HMP-PP and HET-P to produce thiamine phosphate, which is phosphorylated by thiamine monophosphate kinase (ThiL) to produce thiamine pyrophosphate [64].

Strains ATCC 25017 and TX07-7308 contained an operon with six ORFs (thiCOSGDF, Fphi_0086-0090 and F7308_1155-1160, respectively) encoding proteins related to enzymes involved in thiamine biosynthesis in several prokaryotes (Table 3). A similar thiCOSGDF operon was also identified in F. novicida-like strain 3523 [18]. The genetic organization of strains ATCC 25017 and TX07-7308 thiCOSGDF locus was similar to the plasmid-encoded thiCOGE locus involved in thiamine biosynthesis in Rhizobium etli [65]. An analogous gene cluster (thiOGF) is found within plasmid pEA29 of the plant pathogen Erwinia amylovora strain Ea88 [66]. The chromosome of the lithoautotrophic bacterium Ralstonia eutropha $\mathrm{H} 16$ also contains a thiCOSGE locus that is proposed to be involved in de novo synthesis of thiamine [67]. At the protein level, strain ATCC 25017 ThiC was $68 \%$ identical to ThiC of $R$. etli (AAC45972) and Ra. eutropha (H16_A0235), whereas strain ATCC 25017 ThiF was $34 \%$ identical to ThiF of E. amylovora (NP_981993, E-value = 4e-17). Furthermore, ATCC 25017 ThiO and ThiS were 30\% identical to ThiO (H16_A0236) and ThiS (H16_A0237) of Ra. eutropha, respectively $(\mathrm{E}-\mathrm{values}=4 \mathrm{e}-34$ to $2 \mathrm{e}-10)$. Putative thiazole synthase ThiG of strain ATCC 25017 was $~ 51 \%$ identical to ThiG of $R$. etli (AAC45974, E-value $=1 \mathrm{e}-70$ ) and Ra. eutropha (H16_A0238, E-value = 1e-81).

In strains ATCC 25017 and TX07-7308, Fphi_0087 and F7308_1159, respectively, appeared to encode a putative fused protein containing hydroxy-phosphomethylpyrimidine kinase and thiamine-phosphate pyrophosphorylase domains. In some bacteria, these functions are encoded by two different ORFs (thiD and thiE, respectively). Homologs of Fphi_0087 or F7308_1159 were found in several bacteria (e.g., Legionella pneumophila, Coxiella burnetii, Geobacter sulfurreducens, and Colwellia psychrerythraea, $30 \%$ protein identity) and plants (e.g., Arabidopsis thaliana, Zea mays, and Brassica napus, $\sim 29 \%$ protein identity). It has been proposed that these bifunctional enzymes are involved in the synthesis of HMP-PP as well as the condensation of HMP-PP and HET-P to produce thiamine monophosphate $[63,68,69]$. The $5^{\prime}$ untranslated regions of operons involved in thiamine biosynthesis and transport have been shown to contain a regulatory element called THI-box sequence [70]. Based on alignment of conserved sequences upstream of operons involved in thiamine biosynthesis from various bacteria, a putative THI-box sequence (5'-ACCCTTTGAA CCTGATCTAGTTAGCACTAGTGTAGG-3') was identified upstream of thiC in strains TX07-7308 and ATCC 25017. This suggests a thiamine-dependent regulation of thiC in strains ATCC 25017 and TX07-7308, as in other bacteria that have THI-box sequences upstream of TBS genes [63].

In bacteria that lack a TBS pathway, thiamine kinases may facilitate the salvage of dephosphorylated thiamine intermediates from the environment or growth medium [71]. A gene that encoded a putative thiamine pyrophosphokinase (TPK) was found in most members of the genus Francisella, including strains ATCC 25017 and TX07-7308 (Fphi_0159 and F7308_1656, respectively). Strain ATCC 25017 TPK was 27\% identical to Bacillus subtilis TPK (THIN_BACSU, E-value = 1e-10), which catalyzes the direct conversion of thiamine to thiamine pyrophosphate [72]. Thus it appeared that most members of the genus Francisella are capable of salvage of 
Table 3 Identification of genes putatively involved in the biosynthesis of various vitamins and siroheme

\begin{tabular}{|c|c|c|}
\hline $\begin{array}{l}\text { Function } \\
\text { Locus tag (Protein) }\end{array}$ & Annotation & $\begin{array}{l}\text { Closest homolog outside Francisella } \\
\text { locus tag, protein, identity,E-value }\end{array}$ \\
\hline \multicolumn{3}{|c|}{ Thiamine biosynthesis } \\
\hline Fphi_0086 (251 aa) & Thiazole biosynthesis adenylyltransferase (ThiF) & Mrub_1727, 266 aa, 38\%, 7e-41 \\
\hline Fphi_0087 (485 aa) & $\begin{array}{l}\text { Fused protein Phosphomethylpyrimidine kinase (ThiD)/ } \\
\text { Thiamine-phosphate pyrophosphorylase (ThiE) }\end{array}$ & $\operatorname{lpg} 1568,495$ aа, 35\%, 1e-72 \\
\hline Fphi_0088 (259 aа) & Thiazole synthase (ThiG) & $\operatorname{lpg} 1567,263$ aа, 58\%, 2e-86 \\
\hline Fphi_1923 (66 aа) & Thiamine biosynthesis protein (ThiS) & IL0768, 66 aa, 32\%, 1e-04 \\
\hline Fphi_0089 (350 aa) & Thiamine biosynthesis oxidoreductase (ThiO) & Kkor_0127, 351 aa, 34\%, 3e-47 \\
\hline Fphi_0090 (592 aa) & Thiamine biosynthesis protein (ThiC) & CV_0235, 632 aa, 72\%, 0.0 \\
\hline \multicolumn{3}{|c|}{ Riboflavin biosynthesis } \\
\hline Fphi_0395 (306 aa) & Riboflavin kinase (RibF) & D11S_1924, 308 aа, 47\%, 5e-69 \\
\hline Fphi_0713 (356 aa) & Riboflavin biosynthesis protein (RibD) & Acear_1431, 371 aa, 44\%, 5e-79 \\
\hline Fphi_0714 (201 aa) & Riboflavin synthase, alpha subunit (RibC/RibE) & CLD_1677, 228 aa, 48\%, 5e-41 \\
\hline Fphi_0715 (403 аa) & GTP cyclohydrolase \|I (RibA) & DEFDS_1098, 405 aа, 49\%, 5e-104 \\
\hline Fphi_0716 (147 aa) & 6,7-dimethyl-8-ribityllumazine synthase (RibH) & $\operatorname{lpg} 1180,155$ aa, 56\%, 3e-39 \\
\hline \multicolumn{3}{|l|}{ Folate biosynthesis } \\
\hline Fphi_0420 (282 aa) & Methenyltetrahydrofolate cyclohydrolase (FolD) & SULAZ_0428, 284 aa, 60\%, 3e-87 \\
\hline Fphi_0547 (394 aа) & Dihydrofolate synthase (FolC) & Ssed_1653, 421 aa, 34\%, 2e-45 \\
\hline Fphi_0600 (165 aa) & Dihydrofolate reductase (FolA) & BMD_4044, 161 aa, 54\%, 7e-41 \\
\hline Fphi_1791 (184 aa) & Para-aminobenzoate synthase, amidotransferase component & Slin_0655, 205 aa, 38\%, 4e-37 \\
\hline Fphi_1792 (587 aa) & Para-aminobenzoate synthase, aminase component & Csal_2692, 629 aa, 37\%, 3e-99 \\
\hline Fphi_1794 (117 aa) & Dihydroneopterin aldolase (FolB) & WPa_0696, 125 aa, 32\%, 1e-09 \\
\hline Fphi_1795 (421 aа) & Dihydropteroate synthase (FolKP) & RBE_0032, 438 aa, 35\%, 4e-48 \\
\hline \multicolumn{3}{|l|}{ Biotin biosynthesis } \\
\hline Fphi_1798 (428 aa) & $\begin{array}{l}\text { Adenosylmethionine-8-amino-7-oxononanoate } \\
\text { aminotransferase (BioA) }\end{array}$ & CBU_1008, 442 aa, 51\%, 4e-121 \\
\hline Fphi_1799 (313 aа) & Biotin synthase (BioB) & CBU_1007, 321 aa, 61\%, 7e-103 \\
\hline Fphi_1800 (372 aa) & 8-amino-7-oxononanoate synthase (BioF) & Sde_3138, 397 aa, 38\%, 6e-60 \\
\hline Fphi_1801 (244 aа) & Biotin biosynthesis protein (BioC) & CBU_1004, 248 aa, 28\%, 2e-10 \\
\hline Fphi_1803 (226 aа) & Dethiobiotin synthase (BioD) & Sde_3135, 230 aa, 38\%, 5e-37 \\
\hline \multicolumn{3}{|c|}{ Siroheme biosynthesis } \\
\hline Fphi_0284 (323 aa) & Porphobilinogen synthase (HemB) & Amet_0062, 324 aa, 60\%, 1e-110 \\
\hline Fphi_0603 (469 aа) & Glutamyl-tRNA synthetase (GltX) & Fbal_2571, 470 aa, 60\%, 2e-163 \\
\hline Fphi_0691 (300 aа) & Porphobilinogen deaminase (HemC) & APL_1010, 309 aa, 56\%, 4e-86 \\
\hline Fphi_0945 (344 aa) & Uroporphyrinogen decarboxylase (HemE) & Rmag_1026, 347 aa, 50\%, 1e-97 \\
\hline Fphi_1071 (414 aa) & Glutamyl-tRNA reductase (HemA) & PSPA7_5315, 422 aa, 35\%, 2e-70 \\
\hline Fphi_1313 (252 aa) & Uroporphyrinogen-III synthase (HemD) & PSM_A0104, 613 aa, 31\%, 1e-08 \\
\hline Fphi_1400 (400 aa) & Protoporphyrinogen oxidase (HemY/HemG) & Cphamn1_2137, 396 aa, 34\%, 2e-58 \\
\hline Fphi_1812 (433 aа) & Glutamate-1-semialdehyde aminotransferase (HemL) & APJL_1583, 426 aa, 61\%, 2e-151 \\
\hline Fphi_1842 (308 aa) & Coproporphyrinogen III oxidase (HemF) & Tcr_0017, 323 aa, 59\%, 2e-106 \\
\hline Fphi_1900 (338 aa) & Ferrochelatase, protoheme ferro-lyase $(\mathrm{HemH})$ & SMc04019, 342 aа, 50\%, 7e-92 \\
\hline
\end{tabular}


dephosphorylated thiamine intermediates, but some strains (e.g., ATCC 25017 and TX07-7308) can synthesize thiamine de novo, when thiamine intermediates are not available in their environments.

Vitamin $B_{2}$ (riboflavin) is the precursor of coenzymes flavin mononucleotide (FMN) and flavin adenine dinucleotide (FAD), which are cofactors for several biochemical reactions [73]. Most bacteria, fungi, and plants can synthesize riboflavin de novo using one molecule of GTP and two molecules of ribulose 5-phosphate as substrates [74]. Strains ATCC 25017 and TX07-7308 contained five genes encoding enzymes putatively involved in riboflavin biosynthesis, of which four (Fphi_07130716) were within a single locus (Table 3 ). Tetrahydrofolate participates in a number of biochemical reactions and reduced folate cofactors are required for the biosynthesis of a variety of molecules in both prokaryotes and eukaryotes [75,76]. The production of folate involves several enzymes catalyzing the pterin and paraaminobenzoic acid branches of the pathway [77]. Strains ATCC 25017 and TX07-7308 contained seven genes encoding enzymes putatively involved in folate biosynthesis, of which four (Fphi_1791-1795) were clustered together (Table 3).

Vitamin $\mathrm{H}$, commonly known as biotin, acts as a coenzyme in several enzyme-catalyzed carboxylation and decarboxylation reactions [78]. Whereas most bacteria can synthesize biotin de novo using pimelic acid as a precursor, some others have evolved mechanisms for importing this essential cofactor from their natural environments $[79,80]$. In the classic bioABFCD operon of $E$. coli, the bio $A$ and bioBFCD genes are divergently transcribed and encode the enzymes catalyzing the biosynthesis of biotin $[81,82]$. The chromosomes of strains ATCC 25017 and TX07-7308 contained a cluster of genes that resembled the bioABFCD operon of E. coli (Fphi_1798-1803 and F7308_1318- 1314, respectively, Table 3). The bioABFCD operons of strains ATCC 25017 and TX07-7308 were adjacent to the genes encoding enzymes putatively involved in folate biosynthesis.

Environmental bacteria utilize a variety of redox molecules such as porphyrins and other modified tetrapyrroles like heme, siroheme, and adenosylcobalamin for catalysis, energy transfer, and signal transduction [83]. These tetrapyrroles are synthesized de novo using a branched pathway and aminolevulinic acid as the precursor $[84,85]$. Glutamyl-tRNA synthetase, glutamyl-tRNA reductase, and glutamate-1-semialdehyde aminotransferase are involved in the synthesis of aminolevulinic acid using glutamate as the substrate. Two molecules of aminolevulinic acid are condensed by the action porphobilinogen synthase to form porphobilinogen. Four molecules of porphobilinogen are polymerized by the action of porphobilinogen deaminase to form the tetrapyrrole hydroxymethylbilane. Uroporphyrinogen III methyltransferase cyclizes hydroxymethylbilane to produce uroporphyrinogen III. Siroheme synthase, the last enzyme in the pathway, transforms uroporphyrinogen III into siroheme. Strains ATCC 25017 and TX07-7308 contained genes encoding putative homologs of some of the enzymes mentioned above (Table 3).

Most of the publicly available $F$. tularensis genomes contained genes/loci putatively involved in the biosynthesis of riboflavin, folate, biotin, and siroheme (data not shown). The occurrence of these genes/loci in almost all Francisella genomes suggests that these functions are essential for survival in their natural environments. However, the genomes of $F$. tularensis strains conspicuously lacked genes for the biosynthesis of thiamine. It is possible that these strains lost the ancestral TBS genes as a consequence of host adaptation and reductive evolution. The TBS genes identified in this study could be useful in rescuing the thiamine auxotrphy of $F$. tularensis strains and other bacteria in the laboratory. Furthermore, it was evident from comparative genome analyses that strains ATCC 25017 and TX07-7308 as well as most strains of F. tularensis lacked the vitamin $\mathrm{B}_{12}$ biosynthetic pathway. Thus it appears that most extant members of the genus Francisella are vitamin $\mathrm{B}_{12}$ auxotrphs and may require its supplementation for in vitro growth.

\section{Genetics of polysaccharide biosynthesis}

The lipopolysaccharide (LPS) of Francisella spp. has several unique features and has been demonstrated to undergo antigenic variation [86]. The biochemical and immunobiological properties of LPS from several $F$. tularensis strains are well characterized and their LPS is implicated in pathogenesis $[87,88]$. F. philomiragia has been proposed to contain lipooligosaccharide and the lipid A of strain ATCC 25015 has been shown to be rich in shorter fatty acid chains in comparison to F. tularensis [89-91]. The wbt gene cluster of F. tularensis strain Schu S4 (17,378 bp; 31\% GC) is involved in LPS biosynthesis and contained 15 ORFs [92]. In contrast, a similar gene cluster of $F$. novicida strain U112 (13,880 bp; 30.6\% GC) contained only 12 ORFs [92]. The LPS O-antigens of $F$. novicida and $F$. tularensis have been shown to be structurally and immunologically distinct, due in part to the differences in $w b t$ genes involved in their biosynthesis [92,93].

Comparative genomic analyses revealed that strains ATCC 25017 and TX07-7308 contained a cluster of 28 (29,152 bp; $32 \%$ GC) and 42 (44,624 bp; $31.56 \%$ GC) ORFs, respectively, that were related to the $w b t$ gene clusters of F. tularensis strain Schu S4 and F. novicida strain U112. Additional file 2: Table S2 contains a comprehensive list of the annotated ORFs found in the $w b t$ gene clusters of these bacteria. Although strains Schu S4 and U112 contained 
manB and manC ORFs encoding proteins putatively involved in mannose modification adjacent to the $w b t$ gene cluster, strains ATCC 25017 and TX07-7308 contained only a $\operatorname{manB}$ ORF. Among the other genes found in the $w b t$ gene cluster of $F$. tularensis strain Schu S4, only FTT_1450c, FTT_1451c, and FTT_1462c-1464c had homologs among strains ATCC 25017 and TX07-7308. The wbt gene cluster of strain ATCC 25017 contained 7 genes (Fphi_1248, Fphi_1254, Fphi_1255, Fphi_1257-1259, and Fphi_1261) that had no homologs in strains TX077308, Schu S4, and U112. Similalry, the wbt gene cluster of strain TX07-7308 contained 20 genes that had no homologs in strains ATCC 25017, Schu S4, and U112 (F7308_0851-0858, F7308_0860-0864, F7308_0866-0868, and F7308_0872-0875). In contrast, the wbt gene cluster of strain Schu S4 contained four genes that had no homologs in strains ATCC 25017, TX07-7308, and U112 (FTT_1452c-1454c and FTT_1458c), whereas the wbt gene cluster of strain U112 contained six genes that had no homologs in strains ATCC 25017, TX07-7308, and Schu S4 (FTN_1420, FTN_1422, FTN_1424, FTN_1428-1430).

The wbt gene clusters of strains ATCC 25017 and TX07-7308 contained groups of two (Fphi_1246-1247 and F7308_0843-0844, Fphi_1251-1252 and F7308_08470848), three (Fphi_1262-1264 and F7308_0869-0871), and five (Fphi_1265-1269 and F7308_0876-0880) contiguous orthologous ORFs that had no homologs in F. tularensis strain Schu S4 and F. novicida strain U112. They also contained three non-contiguous ORFs (Fphi_1249/F7308_ 0845, Fphi_1256/F7308_0865, and Fphi_1260/F7308_0859) within their wbt gene clusters that had no homologs in strains Schu S4 and U112. The wbt gene clusters of strains Schu S4 and U112 contained three contiguous orthologous ORFs (FTT_1459c-1461c and FTN_1425-1427) that had no homologs in strains ATCC 25017 and TX07-7308. Furthermore, the wbt gene cluster of strain U112 contained a copy of ISFtu 3 transposase (233 aa) that appeared to have truncated the ORF encoding a putative dTDP-D-glucose 4,6-dehydratase (FTN_1420c, WbtM) and the wbt gene cluster of strain Schu S4 contained ISFtu1 transposases (126 aa each). However, the wbt gene clusters of strains ATCC 25017 and TX07-7308 were devoid of transposase genes within or adjacent to them (Additional file 2: Table S2).

From genome comparisons, it was apparent that the wbt gene clusters of strains ATCC 25017, TX07-7308, F. tularensis strain Schu S4, and F. novicida strain U112 display a cassette/mosaic structure with an outer conserved region and an inner variable region. Since a similar cassette/mosaic structure with an outer conserved region and an inner variable region was also observed among the wbt gene clusters of $F$. novicida-like strains Fx1 and 3523 [18], it can be hypothesized that genes in the outer region encode functions common to all strains whereas genes in the inner region encode strain-specific functions. If the number of genes in the inner variable region is an indicator of the complexity of LPS, then the LPS of strains ATCC 25017 and TX07-7308 is likely more different from that of strains Schu S4 and U112. A similar chimeric arrangement has been observed in the gene clusters encoding polysaccharide antigens in Salmonella enterica and it has been proposed that genes in the outer conserved region mediate the conspecific exchange of genes in the inner variable region [94].

Biosynthesis of polysaccharides requires several glycosyltransferases (GTs), which catalyze the transfer of sugars from an activated donor to an acceptor molecule and are usually specific for the glycosidic linkages created [95]. The genomes of strains ATCC 25017, TX077308, Schu S4, and U112 contained yet another cluster of 10-14 ORFs oriented in the same direction that encoded putative GTs and other proteins related to enzymes involved in polysaccharide biosynthesis and/or cell wall/membrane biogenesis. Additional file 3: Table S3 contains a list of the annotated ORFs found within this gene cluster. A similar gene cluster, which was tentatively designated psl (polysaccharide synthesis locus), was also identified in F. novicida-like strains Fx1 and 3523 [18]. Whereas strain ATCC 25017 had 9 strainspecific ORFs (Fphi_1471-1473 and Fphi_1475-1480) within the psl cluster, strain TX07-7308 contained only two (F7308_1111 and F7308_1118) strain-specific ORFs within this cluster. Furthermore, strain Schu S4 contained 3 strain-specific ORFs (FTT_0794-0796) within the psl cluster and strain U112 contained only one (FTN_1216) strain-specific ORF within this cluster. These strain-specific ORFs were flanked by a single orthologous ORF encoding a putative HAD family hydrolase (FTT_0800/Fphi_1481/F7308_1119/FTN_1211) and a sugar transferase (FTT_0790/Fphi_1468/F7308_1110/ FTN_1220). Based on gene content and organization, it may be surmised that the psl gene cluster was involved in LPS and/or exopolysaccharide (EPS) biosynthesis. Since the psl gene cluster of strain ATCC 25017 contained more genes compared to strains TX07-7308, Schu S4, and U112, it is possible that the LPS/EPS of this strain is more complex.

\section{Atypical arsenic resistance loci}

Arsenic is an environmental pollutant and some microorganisms have evolved mechanisms of resistance to this cytotoxic agent. Arsenic exists in two oxidation states, arsenite (AsIII) and arsenate (AsV), in biological systems [96]. In most bacteria, the minimal arsenical resistance operon contains three ORFs $(\operatorname{ars} R B C)$ wherein the conversion of arsenate to arsenite is accomplished by a reductase (product of arsC), arsenite is transported out of the cell by a membrane-bound efflux pump (product of 
$\operatorname{ars} B)$, and $\operatorname{ars} R$ encodes an arsenic-resistance regulatory protein. Plasmid or transposon-mediated horizontal transfer of genes that confer arsenic resistance has been well documented [96].

Strain ATCC 25017 contained a locus encoding a truncated arsenite/antimonite exporter (Fphi_1745, arsB, 27 aa), an arsenate reductase (Fphi_1746, arsC, 139 aa), a sulfate permease (Fphi_1747, 526 aa), a hypothetical protein (Fphi_1748, 111 aa), and a mechanosensitive ion channel family protein (Fphi_1749, 277 aa). This locus was adjacent to the one that encoded several integrative/ transposable elements (Fphi_1740-1744; Figure 4). Whereas homologs of Fphi_1746 and Fphi_1748 were absent in all other publicly available complete Francisella genomes, homologs of Fphi_1747 and Fphi_1749 were found in strain TX07-7308 (F7308_0311, 408 aa and F7308_1369, 398 aa, respectively). Strain ATCC 25017 contained yet another locus encoding putative arsenical resistance proteins (Fphi_1817-1818; Figure 4). Strain TX07-7308 contained an orthologous arsenical resistance locus (F7308 1298-1299; Figure 4). At the predicted protein level, Fphi_1818 (arsB, 342 aa) and Fphi_1817 (arsR, 112 aa) had 97\% identities to F7308_1298 (342 aa) and F7308_1299 (110 aa), respectively. Strain TX07-7308 contained yet another locus encoding putative arsenical resistance proteins (F7308_0309-0310; Figure 4). At the predicted protein level, Fphi_1818 and Fphi_1817 had 77\% and 52\% identities to F7308_0310 (arsB, 342 aa) and F7308_0309 (arsR, 113 aa), respectively.

An arsenical resistance locus was also found in $F$. novicida strain U112 (FTN_0800 and FTN_0801, 95\% and 85\% identity to Fphi_1818 and Fphi_1817, respectively). Although a gene encoding a putative IS4 family transposase $(247$ aa) was found adjacent to the $\operatorname{ars} R B$ locus of strain U112, no such gene was found near the orthologous arsRB loci of strains ATCC 25017 and TX07-7308. Furthermore, at the predicted protein level, strain ATCC 25017 ArsB (Fphi_1818) was 61\% identical to the arsenite efflux transporter of Bacillus subtilis (BSU25790, 346 aa) and ArsR (Fphi_1817) was 37\% identical to ArsR repressor of B. subtilis (BSU25810, 105 aa). Although BSU25790 and BSU25810 have been shown to be involved in arsenate and arsenite resistance in B. subtilis [97], biochemical characterization is required to ascertain the role of the putative arsenical resistance loci of Francisella strains. In addition, adjacent to Fphi_1817-1818, strain ATCC 25017 contained a gene encoding a putative protein (Fphi_1819, 109 aa) that was $42 \%$ identical to the small multidrug resistance antiporter (EmrE) of E. coli. Interestingly, none of the $F$. tularensis genomes sequenced to date contained homologs of arsRB loci or emrE. The occurrence of an ortho$\log$ of emrE in strains U112 (FTN_0799, 109 aa) and TX07-7308 (F7308_1297, 109 aa) suggests that arsRB and $e m r E$ loci are ancestral and evolutionarily conserved among some environmental lineages of the genus Francisella.

\section{Summary of important genetic traits and phylogenetic analyses}

Based on whole genome comparisons of different strains of Francisella, the following genetic traits are obvious. Whereas strain TX07-7308 lacked the lac operon, strain ATCC 25017 lacked genes for GlcUA utilization. However, strains ATCC 25017 and TX07-7308 contained genes for thiamine biosynthesis. Although Type A and Type B strains of $F$. tularensis had lost loci for GlcUA

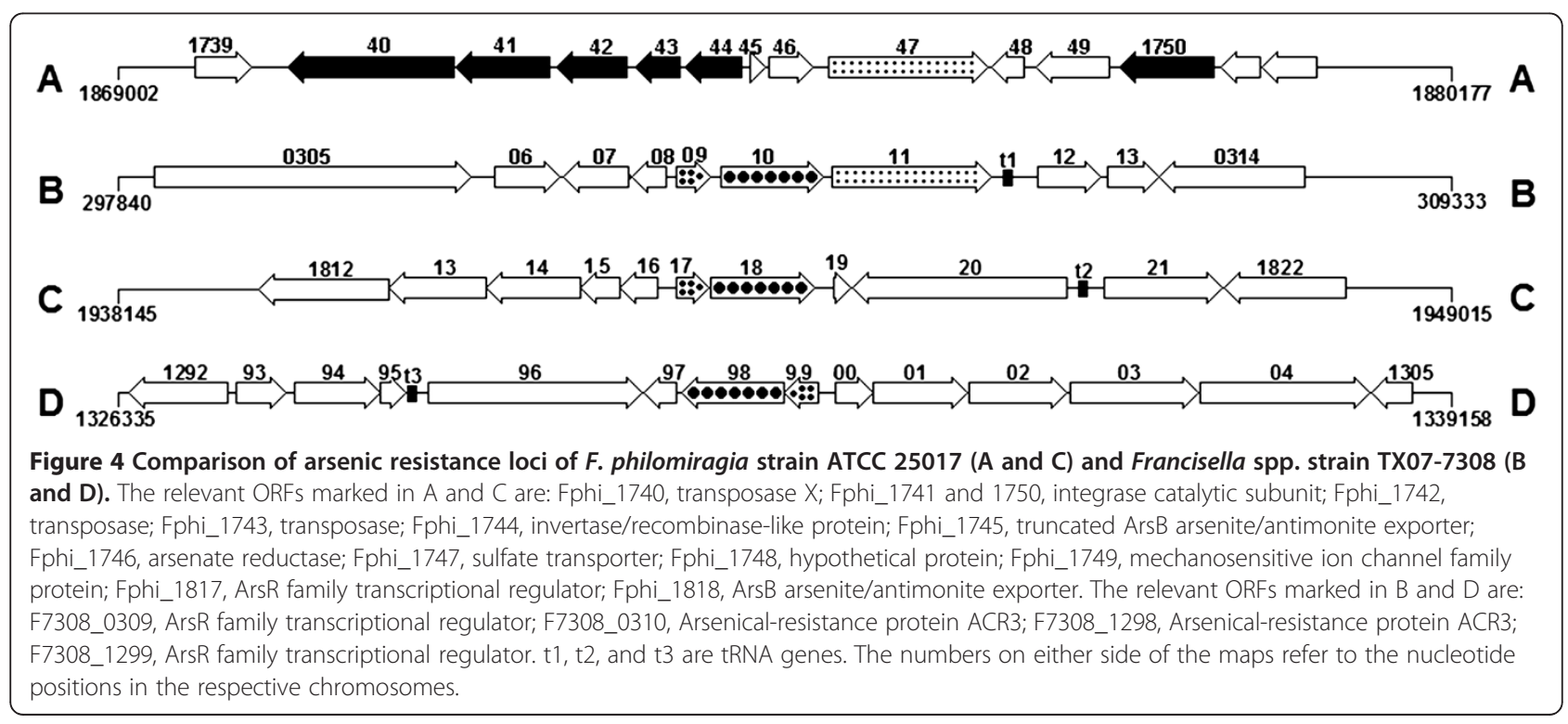


utilization and thiamine biosynthesis, they do contain a vestigial lac $Z$ gene.

While F. novicida-like strain Fx1 contained the lac operon in addition to genes for GlcUA utilization, F. novicida-like strain 3523 contained the lac operon along with GlcUA utilization and thiamine biosynthesis genes [18]. Notably, F. novicida strain U112 lacked genes/loci for GlcUA utilization, thiamine biosynthesis, and lactose utilization. However, strain U112 contained an $\operatorname{arsRB}$ locus, which was absent in strains 3523 and Fx1 [18], but was present in strains ATCC 25015, ATCC 25017, and TX07-7308. It is possible that strain U112, an environmental isolate of $F$. novicida, had retained an $\operatorname{ars} R B$ locus, but had lost loci for lactose and GlcUA utilization as well as genes for thiamine biosynthesis because of niche selection. Similarly, extant strains of $F$. tularensis appeared to have lost the arsRB locus, loci for lactose and GlcUA utilization, in addition to genes for thiamine biosynthesis as a consequence of host adaptation and reductive evolution. Based on comparative genome analyses, it may be concluded that the genes/loci encoding putative phenotypes such as arsenite resistance, lactose utilization, biosynthesis of various vitamins/cofactors, type IV pili, oligopeptide $A B C$ transporter system, and iron metabolism are more ancient within the genus Francisella. Figure 5A summarizes some of the genetic traits among Francisella strain TX07-7308, F. philomiragia strains ATCC 25015 and ATCC 25017, F. novicidalike strains 3523 and Fx1, F. novicida strain U112, and $F$. tularensis strain Schu S4.

Several previous phylogenetic analyses based on the $16 \mathrm{~S}$ RNA gene have indicated that $F$. philomiragia is more ancient than F. tularensis $[98,99]$. Analyses based on $\sim 8,000$ single nucleotide polymorphisms across the genomes have demonstrated a relatively large evolutionary separation of
F. philomiragia from F. tularensis [100]. Furthermore, pairwise analyses of multiple Francisella genomes have indicated that the average nucleotide identity between $F$. tularensis and F. philomiragia ranges between $80.6 \%$ and 81.2\% [20]. Since deletion-based phylogenetic analyses have demonstrated that $F$. novicida is ancient to $F$. tularensis and that both acquisition and loss of genes have occurred during the evolution of different species within the genus Francisella [101], it is possible that F. novicida represents an intermediate environmental and/or pathogenic lineage between $F$. philomiragia and F. tularensis. Phylogenetic analyses based on full-length 16S rRNA and $s d h A$ genes support these observations (Figure $5 \mathrm{~B}$ and $\mathrm{C}$ ).

\section{Conclusions}

Analyses of the genomes of strains ATCC 25017 and TX07-7308 imply that these strains are metabolically versatile and they represent new links in the chain of evolution from an early ancestor to the extant strains of F. novicida and F. tularensis. Although strains ATCC 25017 and TX07-7308 were isolated in different parts of the USA, both were associated with aquatic environments and their recent common ancestry was evident from genome comparisons. It was also evident that strains of $F$. tularensis have lost several ancestral traits and the consequent reductive evolution may be a cause for as well as an effect of niche selection of these strains. It is likely that the progenitor of $F$. tularensis strains originated in an aquatic environment and became host-adapted during subsequent evolution. Although numerous previous studies have focused on the genetic relationships and evolutionary contexts among $F$. novicida and $F$. tularensis strains, comparative genome sequence analyses of $F$. philomiragia, $F$. novicida, and F. tularensis strains have provided a comprehensive

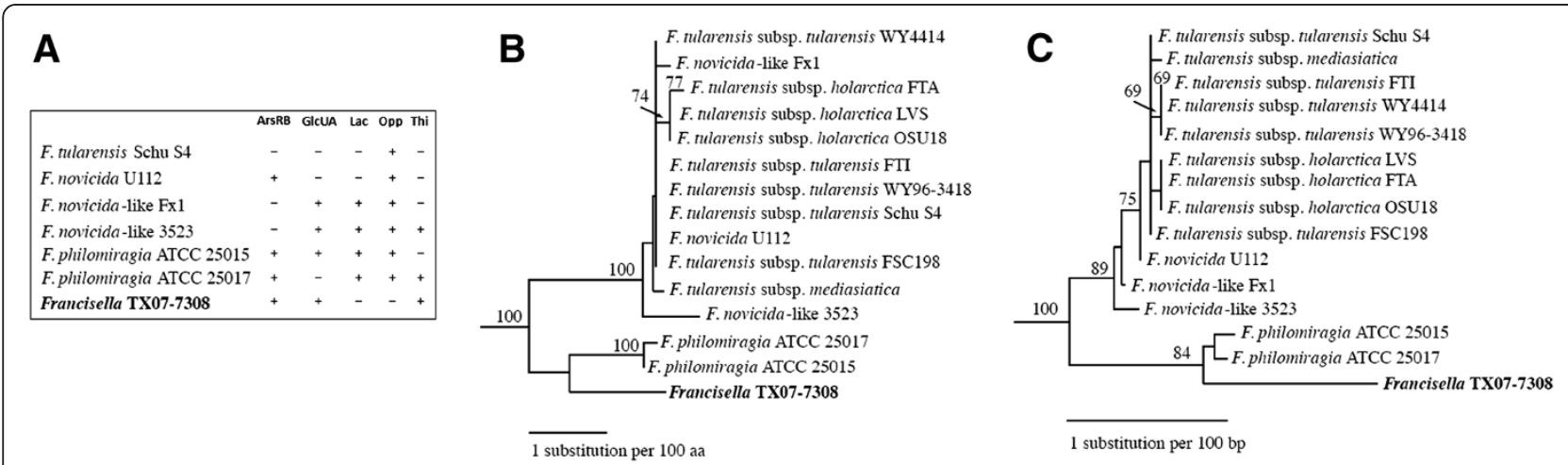

Figure 5 A Summary of important genetic traits among strains TX07-7308, ATCC 25015, ATCC 25017, 3523, Fx1, U112, and Schu S4. ArsRB. Arsenic resistance locus, GIcUA. Glucuronate metabolism locus, Lac. Lactose metabolism locus, Opp. Oligopeptide ABC transporter locus, Thi. Thiamine biosynthesis locus. + indicates the presence and - indicates the absence of a particualr locus in each strain. B. Neighbor joining tree using succinate dehydrogenase (sdhA) genes. Phylogenetic relationships among strains TX07-7308, ATCC 25015, ATCC 25017, U112, and eleven other Francisella isolates based on SdhA sequences are shown. Nodes with bootstrap support greater than $70 \%$ are indicated. C. As in Figure 2B, but using full-length 165 rRNA genes. 
account of the innate and acquired genetic traits among this important group of bacteria.

\section{Additional files}

Additional file 1: Table S1. Characteristics of putative integrative and transposable elements. This table contains a list of the various putative integrative and transposable elements identified in Francisella strain TX077308 and F. philomiragia strain ATCC 25017.

Additional file 2: Table S2. Comparison of the wbt gene clusters of four different strains within the genus Francisella. This table contains data related to the comparison of the $w b t$ gene clusters of strains TX07-7308, ATCC 25017, U112, and Schu S4.

Additional file 3: Table S3. Comparison of the psl gene clusters of four different strains within the genus Francisella. This table contains data related to the comparison of the ps/ gene clusters of strains TX07-7308, ATCC 25017, U112, and Schu S4.

\section{Competing interests}

The authors state that there are no financial or non-financial competing interests associated with this manuscript.

\section{Authors' contributions}

SS performed the annotation using RAST, planned the comparative analysis, and drafted most of the manuscript. JFC and JMP contributed to whole genome comparisons. SP and CRK conceived the study, participated in genome analyses, and helped draft the manuscript. All authors read and approved the final manuscript.

\section{Acknowledgements}

This study was funded by the United States Department of Homeland Security (Chemical and Biological Division, Science and Technology Directorate) through an Interagency Agreement (grant number HSHQDC-08$\mathrm{X}$-00790) and by the Centers for Disease Control and Prevention. The authors wish to thank Ms. Karen Davenport and other members of the Genome Sequencing and Finishing Team at the Los Alamos National Laboratory for help with sequencing strain TX07-7308

\section{Author details}

${ }^{1}$ Bioscience Division, Los Alamos National Laboratory, Los Alamos, NewMexico 87545, USA. ²Division of Vector-Borne Infectious Diseases, Centers for Disease Control and Prevention, Fort Collins, Colorado 80521, USA. ${ }^{3}$ Chemical and Biological Division, Science and Technology Directorate, Department of Homeland Security, Washington, DC, USA.

Received: 7 December 2011 Accepted: 14 August 2012 Published: 24 August 2012

\section{References}

1. Jensen WI, Owen CR, Jellison WL: Yersinia philomiragia sp. n., a new member of the Pasteurella group of bacteria, naturally pathogenic for the muskrat (Ondatra zibethica). J Bacteriol 1969, 100(3):1237-1241.

2. Ursing J, Steigerwalt A, Brenner D: Lack of genetic relatedness between Yersinia philomiragia (the "Philomiragia" bacterium) and Yersinia species. Curr Microbiol 1980, 4(4):231-233.

3. Wenger JD, Hollis DG, Weaver RE, Baker CN, Brown GR, Brenner DJ, Broome CV: Infection caused by Francisella philomiragia (formerly Yersinia philomiragia). A newly recognized human pathogen. Ann Intern Med 1989, 110(11):888-892.

4. Hollis DG, Weaver RE, Steigerwalt AG, Wenger JD, Moss CW, Brenner DJ: Francisella philomiragia comb. nov. (formerly Yersinia philomiragia) and Francisella tularensis biogroup novicida (formerly Francisella novicida) associated with human disease. J Clin Microbiol 1989, 27(7):1601-1608.

5. Clarridge JE, Raich TJ, Sjosted A, Sandstrom G, Darouiche RO, Shawar RM, Georghiou PR, Osting C, Vo L: Characterization of two unusual clinically significant Francisella strains. J Clin Microbiol 1996, 34(8):1995-2000.

6. Broman T, Thelaus J, Andersson AC, Backman S, Wikstrom P, Larsson E, Granberg M, Karlsson L, Back E, Eliasson H, et al: Molecular detection of persistent Francisella tularensis subspecies holarctica in natural waters. Int J Microbiol 2011, 2011:10. 2011(Article ID 851946):10 pages.

7. Ender PT, Dolan MJ: Pneumonia associated with near-drowning. Clin Infect Dis 1997, 25(4):896-907.

8. Friis-Moller A, Lemming LE, Valerius NH, Bruun B: Problems in identification of Francisella philomiragia associated with fatal bacteremia in a patient with chronic granulomatous disease. J Clin Microbiol 2004, 42(4):1840-1842.

9. Sicherer SH, Asturias EJ, Winkelstein JA, Dick JD, Willoughby RE: Francisella philomiragia sepsis in chronic granulomatous disease. Pediatr Infect Dis J 1997, 16(4):420-422

10. Polack FP, Harrington SM, Winkelstein JA, Merz WG, Willoughby RE: Recurrent Francisella philomiragia sepsis in chronic granulomatous disease. Pediatr Infect Dis J 1998, 17(5):442-443.

11. Mailman $\mathrm{TL}$, Schmidt MH: Francisella philomiragia adenitis and pulmonary nodules in a child with chronic granulomatous disease. Can J Infect Dis Med Microbiol 2005, 16(4):245-248.

12. Cora MC, Neel JA, Tarigo J, Post K, Barnes J: Francisella philomiragia septicemia in a dog. J Vet Intern Med 2010, 24(4):969-972.

13. Petersen JM, Carlson J, Yockey B, Pillai S, Kuske C, Garbalena G, Pottumarthy $S$, Chalcraft L: Direct isolation of Francisella spp. from environmental samples. Lett Appl Microbiol 2009, 48(6):663-667.

14. Schrallhammer M, Schweikert M, Vallesi A, Verni F, Petroni G: Detection of a novel subspecies of Francisella noatunensis as endosymbiont of the ciliate Euplotes raikovi. Microb Ecol 2011, 61(2):455-464.

15. Berrada ZL, Telford SR 3rd: Diversity of Francisella species in environmental samples from Martha's Vineyard, Massachusetts. Microb Ecol 2009, 59(2):277-283

16. Ottem KF, Nylund A, Karlsbakk E, Friis-Moller A, Kamaishi T: Elevation of Francisella philomiragia subsp. noatunensis Mikalsen et al. (2007) to Francisella noatunensis comb. nov. [syn. Francisella piscicida Ottem et al. (2008) syn. nov.] and characterization of Francisella noatunensis subsp. orientalis subsp. nov., two important fish pathogens. J Appl Microbiol 2009, 106(4):1231-1243.

17. Rohmer L, Fong C, Abmayr S, Wasnick M, Larson Freeman TJ, Radey M, Guina T, Svensson K, Hayden HS, Jacobs M, et al: Comparison of Francisella tularensis genomes reveals evolutionary events associated with the emergence of human pathogenic strains. Genome Biol 2007, 8(6):R102.

18. Siddaramappa S, Challacombe JF, Petersen JM, Pillai S, Hogg G, Kuske CR: Common ancestry and novel genetic traits of Francisella novicida-like isolates from North America and Australia as revealed by comparative genomic analyses. App/ Environ Microbio/ 2011, 77(15):5110-5122.

19. Champion MD, Zeng Q, Nix EB, Nano FE, Keim P, Kodira CD, Borowsky M, Young $S$, Koehrsen M, Engels $R$, et al: Comparative genomic characterization of Francisella tularensis strains belonging to low and high virulence subspecies. PLoS Pathog 2009, 5(5):e1000459.

20. Larsson P, Elfsmark D, Svensson K, Wikstrom P, Forsman M, Brettin T, Keim P, Johansson A: Molecular evolutionary consequences of niche restriction in Francisella tularensis, a facultative intracellular pathogen. PLOS Pathog 2009, 5(6):e1000472.

21. Brevik OJ, Ottem KF, Nylund A: Multiple-locus, variable number of tandem repeat analysis (MLVA) of the fish-pathogen Francisella noatunensis. BMC Vet Res 2011, 7:5

22. Molins CR, Carlson JK, Coombs J, Petersen JM: Identification of Francisella tularensis subsp. tularensis $A 1$ and $A 2$ infections by real-time polymerase chain reaction. Diagn Microbiol Infect Dis 2009, 64(1):6-12.

23. Woyke T, Xie G, Copeland A, Gonzalez JM, Han C, Kiss H, Saw JH, Senin P, Yang C, Chatterji S, et al: Assembling the marine metagenome, one cell at a time. PLoS One 2009, 4(4):e5299.

24. Barabote RD, Xie G, Brettin TS, Hinrichs SH, Fey PD, Jay JJ, Engle JL, Godbole $\mathrm{SD}$, Noronha JM, Scheuermann RH, et al: Complete genome sequence of Francisella tularensis subspecies holarctica FTNF002-00. PLoS One 2009, 4(9):e7041.

25. Beckstrom-Sternberg S, Auerbach R, Godbole S, Pearson J, BeckstromSternberg J, Deng Z, Munk C, Kubota K, Zhou Y, Bruce D, et al: Complete genomic characterization of a pathogenic A.ll strain of Francisella tularensis subspecies tularensis. PLoS One 2007, 2(9):e947.

26. Aziz RK, Bartels D, Best AA, DeJongh M, Disz T, Edwards RA, Formsma K, Gerdes S, Glass EM, Kubal M, et al: The RAST Server: rapid annotations using subsystems technology. BMC Genomics 2008, 9:75.

27. Dereeper A, Guignon V, Blanc G, Audic S, Buffet S, Chevenet F, Dufayard JF, Guindon S, Lefort V, Lescot M, et al: Phylogeny.fr: robust phylogenetic 
analysis for the non-specialist. Nucleic Acids Res 2008, 36(Web Server issue): W465-W469.

28. Edgar RC: MUSCLE: multiple sequence alignment with high accuracy and high throughput. Nucleic Acids Res 2004, 32(5):1792-1797.

29. Tamura K, Nei M, Kumar S: Prospects for inferring very large phylogenies by using the neighbor-joining method. Proc Natl Acad Sci USA 2004, 101(30):11030-11035.

30. Le Pihive E, Blaha D, Chenavas S, Thibault F, Vidal D, Valade E: Description of two new plasmids isolated from Francisella philomiragia strains and construction of shuttle vectors for the study of Francisella tularensis. Plasmid 2009, 62(3):147-157.

31. Lee ZM, Bussema C 3rd, Schmidt TM: rrnDB: documenting the number of rRNA and tRNA genes in bacteria and archaea. Nucleic Acids Res 2009, 37(Database issue):D489-D493.

32. Dethlefsen L, Schmidt TM: Performance of the translational apparatus varies with the ecological strategies of bacteria. J Bacteriol 2007, 189(8):3237-3245.

33. Zahn $\mathrm{K}$, Inui $\mathrm{M}$, Yukawa $\mathrm{H}$ : Structure, expression and products of the ribosomal RNA operons of Rhodopseudomonas palustris No. 7. Mol Genet Genomics 2001, 265(5):778-790.

34. Boyer SL, Flechtner VR, Johansen JR: Is the 16S-23S rRNA internal transcribed spacer region a good tool for use in molecular systematics and population genetics? A case study in cyanobacteria. Mol Biol Evol 2001, 18(6):1057-1069.

35. Thomas R, Johansson A, Neeson B, Isherwood K, Sjostedt A, Ellis J, Titball RW: Discrimination of human pathogenic subspecies of Francisella tularensis by using restriction fragment length polymorphism. J Clin Microbiol 2003, 41(1):50-57.

36. Shi W, Sun H: Type IV pilus-dependent motility and its possible role in bacterial pathogenesis. Infect Immun 2002, 70(1):1-4.

37. Zogaj X, Chakraborty S, Liu J, Thanassi DG, Klose KE: Characterization of the Francisella tularensis subsp. novicida type IV pilus. Microbiology 2008, 154(Pt 7):2139-2150.

38. Gil $H$, Benach $J$, Thanassi DG: Presence of pili on the surface of Francisella tularensis. Infect Immun 2004, 72(5):3042-3047.

39. Hager AJ, Bolton DL, Pelletier MR, Brittnacher MJ, Gallagher LA, Kaul R, Skerrett SJ, Miller SI, Guina T: Type IV pili-mediated secretion modulates Francisella virulence. Mol Microbiol 2006, 62(1):227-237.

40. Salomonsson EN, Forslund A-L, Forsberg A: Type IV pili in Francisella -a virulence trait in an intracellular pathogen. Front Microbio 2011, 2(29). doi:10.3389/fmicb.2011.00029

41. Verhoeven AB, Durham-Colleran MW, Pierson T, Boswell WT, Van Hoek ML: Francisella philomiragia biofilm formation and interaction with the aquatic protist Acanthamoeba castellanii. Biol Bull 2010, 219(2):178-188.

42. Monnet V: Bacterial oligopeptide-binding proteins. Cell Mol Life Sci 2003, 60(10):2100-2114.

43. Taylor DL, Ward PN, Rapier CD, Leigh JA, Bowler LD: Identification of a differentially expressed oligopeptide binding protein (Opp A2) in Streptococcus uberis by representational difference analysis of CDNA. J Bacteriol 2003, 185(17):5210-5219.

44. Titball RW, Petrosino JF: Francisella tularensis genomics and proteomics. Ann N Y Acad Sci 2007, 1105:98-121.

45. Kiss K, Liu W, Huntley JF, Norgard MV, Hansen EJ: Characterization of fig operon mutants of Francisella novicida U112. FEMS Microbiol Lett 2008, 285(2):270-277.

46. Ramakrishnan G, Meeker A, Dragulev B: fsIE is necessary for siderophoremediated iron acquisition in Francisella tularensis Schu S4. J Bacteriol 2008, 190(15):5353-5361

47. Lindgren H, Honn M, Golovlev I, Kadzhaev K, Conlan W, Sjostedt A: The 58-kilodalton major virulence factor of Francisella tularensis is required for efficient utilization of iron. Infect Immun 2009, 77(10):4429-4436.

48. Peekhaus N, Conway T: What's for dinner?: Entner-Doudoroff metabolism in Escherichia coli. J Bacteriol 1998, 180(14):3495-3502.

49. Shulami S, Gat O, Sonenshein AL, Shoham Y: The glucuronic acid utilization gene cluster from Bacillus stearothermophilus T-6. J Bacteriol 1999, 181(12):3695-3704.

50. Brown PM, Caradoc-Davies TT, Dickson JM, Cooper GJ, Loomes KM, Baker EN: Purification, crystallization and preliminary crystallographic analysis of mouse myo-inositol oxygenase. Acta Crystallogr Sect F Struct Biol Cryst Commun 2006, 62(Pt 8):811-813.
51. Michell $\mathrm{RH}$ : Inositol derivatives: evolution and functions. Nat Rev Mol Cell Biol 2008, 9(2):151-161.

52. Neuwald AF, York JD, Majerus PW: Diverse proteins homologous to inositol monophosphatase. FEBS Lett 1991, 294(1-2):16-18.

53. Nigou J, Dover LG, Besra GS: Purification and biochemical characterization of Mycobacterium tuberculosis SuhB, an inositol monophosphatase involved in inositol biosynthesis. Biochemistry 2002, 41(13):4392-4398.

54. Wilson CJ, Zhan H, Swint-Kruse L, Matthews KS: The lactose repressor system: paradigms for regulation, allosteric behavior and protein folding. Cell Mol Life Sci 2007, 64(1):3-16.

55. Coombs JM, Brenchley JE: Biochemical and phylogenetic analyses of a coldactive beta-galactosidase from the lactic acid bacterium Carnobacterium piscicola BA. App/ Environ Microbiol 1999, 65(12):5443-5450.

56. Daniel RA, Haiech J, Denizot F, Errington J: Isolation and characterization of the lacA gene encoding beta-galactosidase in Bacillus subtilis and a regulator gene, lacR. J Bacteriol 1997, 179(17):5636-5638.

57. Guiso N, Ullmann A: Expression and regulation of lactose genes carried by plasmids. J Bacteriol 1976, 127(2):691-697.

58. Leong-Morgenthaler $\mathrm{P}$, Zwahlen MC, Hottinger H: Lactose metabolism in Lactobacillus bulgaricus: analysis of the primary structure and expression of the genes involved. J Bacteriol 1991, 173(6):1951-1957.

59. Stoebel DM: Lack of evidence for horizontal transfer of the lac operon into Escherichia coli. Mol Biol Evol 2005, 22(3):683-690.

60. Cornelis G: Sequence relationships between plasmids carrying genes for lactose utilization. J Gen Microbiol 1981, 124(1):91-97.

61. Gilbert HJ, Hall J: Molecular cloning of Streptococcus bovis lactose catabolic genes. J Gen Microbiol 1987, 133(8):2285-2293.

62. Timoney JF, Taylor DE, Shin S, McDonough P: pJT2: unusual H1 plasmid in a highly virulent lactose-positive and chloramphenicol-resistant Salmonella typhimurium strain from calves. Antimicrob Agents Chemother 1980, 18(3):480-482.

63. Rodionov DA, Vitreschak AG, Mironov AA, Gelfand MS: Comparative genomics of thiamin biosynthesis in procaryotes. New genes and regulatory mechanisms. J Biol Chem 2002, 277(50):48949-48959.

64. Begley TP, Downs DM, Ealick SE, McLafferty FW, Van Loon AP, Taylor S, Campobasso N, Chiu HJ, Kinsland C, Reddick JJ, et al: Thiamin biosynthesis in prokaryotes. Arch Microbiol 1999, 171(5):293-300.

65. Miranda-Rios J, Morera C, Taboada H, Davalos A, Encarnacion S, Mora J, Soberon M: Expression of thiamin biosynthetic genes (thiCOGE) and production of symbiotic terminal oxidase cbb3 in Rhizobium etli. J Bacteriol 1997, 179(22):6887-6893.

66. McGhee GC, Jones AL: Complete nucleotide sequence of ubiquitous plasmid pEA29 from Erwinia amylovora strain Ea88: gene organization and intraspecies variation. Appl Environ Microbiol 2000, 66(11):4897-4907.

67. Pohlmann A, Fricke WF, Reinecke F, Kusian B, Liesegang H, Cramm R, Eitinger T, Ewering C, Potter M, Schwartz E, et al: Genome sequence of the bioplastic-producing "Knallgas" bacterium Ralstonia eutropha H16. Nat Biotechnol 2006, 24(10):1257-1262.

68. Rapala-Kozik M, Olczak M, Ostrowska K, Starosta A, Kozik A: Molecular characterization of the thi3 gene involved in thiamine biosynthesis in Zea mays: CDNA sequence and enzymatic and structural properties of the recombinant bifunctional protein with 4-amino-5-hydroxymethyl-2methylpyrimidine (phosphate) kinase and thiamine monophosphate synthase activities. Biochem J 2007, 408(2):149-159.

69. Kim YS, Nosaka K, Downs DM, Kwak JM, Park D, Chung IK, Nam HG: A Brassica cDNA clone encoding a bifunctional hydroxymethylpyrimidine kinase/thiamin-phosphate pyrophosphorylase involved in thiamin biosynthesis. Plant Mol Biol 1998, 37(6):955-966.

70. Miranda-Rios J: The THI-box riboswitch, or how RNA binds thiamin pyrophosphate. Structure 2007, 15(3):259-265.

71. Jurgenson CT, Begley TP, Ealick SE: The structural and biochemical foundations of thiamin biosynthesis. Annu Rev Biochem 2009, 78:569-603.

72. Melnick J, Lis E, Park JH, Kinsland C, Mori H, Baba T, Perkins J, Schyns G, Vassieva O, Osterman A, et al: Identification of the two missing bacterial genes involved in thiamine salvage: thiamine pyrophosphokinase and thiamine kinase. J Bacterio/ 2004, 186(11):3660-3662.

73. Vitreschak AG, Rodionov DA, Mironov AA, Gelfand MS: Regulation of riboflavin biosynthesis and transport genes in bacteria by transcriptional and translational attenuation. Nucleic Acids Res 2002, 30(14):3141-3151.

74. Fischer $\mathrm{M}$, Bacher $\mathrm{A}$ : Biosynthesis of vitamin B2: structure and mechanism of riboflavin synthase. Arch Biochem Biophys 2008, 474(2):252-265. 
75. Green JM, Nichols BP, Matthews RG: Folate biosynthesis, reduction, and polyglutamylation. In Escherichia coli and Salmonella: cellular and molecular biology. 2nd edition. Edited by Neidhardt FC, Curtiss R III, Ingraham JL, Lin ECC, Low KB, Magasanik B, Reznikoff WS, Riley MA, Schaechter M, Umbarger HE. Washington, D.C: ASM Press; 1996:665-673.

76. Lucock M: Folic acid: nutritional biochemistry, molecular biology, and role in disease processes. Mol Genet Metab 2000, 71(1-2):121-138.

77. de Crecy-Lagard V, El Yacoubi B, de la Garza RD, Noiriel A, Hanson AD: Comparative genomics of bacterial and plant folate synthesis and salvage: predictions and validations. BMC Genomics 2007, 8:245.

78. Moss J, Lane MD: The biotin-dependent enzymes. Adv Enzymol Relat Areas Mol Biol 1971, 35:321-442

79. Schneider $G$, Lindqvist $Y$ : Structural enzymology of biotin biosynthesis. FEBS Lett 2001, 495(1-2):7-11.

80. Rodionov DA, Mironov AA, Gelfand MS: Conservation of the biotin regulon and the BirA regulatory signal in eubacteria and archaea. Genome Res 2002, 12(10):1507-1516.

81. Guha A: Divergent orientation of transcription from the biotin locus of Escherichia coli. J Mol Biol 1971, 56(1):53-62.

82. Cleary PP, Campbell A: Deletion and complementation analysis of biotin gene cluster of Escherichia coli. J Bacteriol 1972, 112(2):830-839.

83. Lascelles J: Synthesis of tetrapyrroles by microorganisms. Physiol Rev 1961, 41:417-441.

84. Warren MJ, Smith AG, Deery E, Rose R-S: Biosynthesis of siroheme and coenzyme F430. In Tetrapyrroles: birth, life and death. Edited by Warren MJ Smith AG. New York: Springer; 2009:344-351.

85. Zappa S, Li K, Bauer CE: The tetrapyrrole biosynthetic pathway and its regulation in Rhodobacter capsulatus. Adv Exp Med Biol 2010, 675:229-250.

86. Gunn JS, Ernst RK: The structure and function of Francisella lipopolysaccharide. Ann N Y Acad Sci 2007, 1105:202-218.

87. Clay CD, Soni S, Gunn JS, Schlesinger LS: Evasion of complementmediated lysis and complement C3 deposition are regulated by Francisella tularensis lipopolysaccharide 0 antigen. J Immunol 2008, 181(8):5568-5578.

88. Kieffer TL, Cowley S, Nano FE, Elkins KL: Francisella novicida LPS has greater immunobiological activity in mice than F. tularensis LPS, and contributes to F. novicida murine pathogenesis. Microbes Infect 2003, 5(5):397-403.

89. Onoprienko NN, Pavlovich NV: The role of lipopolysaccharide in toxicity of Francisella genus bacteria. Mol Gen Mikrobiol Virusol 2003, 3:25-28.

90. Maslova NN, Pavlovich NV, Sorokin VM, Zurabian VA: Characterization of structure and antigenic activity of lipopolysaccharides of genus Francisella. Mol Gen Mikrobiol Virusol 1998, 3:26-29.

91. Schilling B, McLendon MK, Phillips NJ, Apicella MA, Gibson BW: Characterization of lipid A acylation patterns in Francisella tularensis, Francisella novicida, and Francisella philomiragia using multiple-stage mass spectrometry and matrixassisted laser desorption/ionization on an intermediate vacuum source linear ion trap. Anal Chem 2007, 79(3):1034-1042.

92. Thomas RM, Titball RW, Oyston PC, Griffin K, Waters E, Hitchen PG, Michell SL, Grice ID, Wilson JC, Prior JL: The immunologically distinct O antigens from Francisella tularensis subspecies tularensis and Francisella novicida are both virulence determinants and protective antigens. Infect Immun 2007, 75(1):371-378.

93. Raynaud C, Meibom KL, Lety MA, Dubail I, Candela T, Frapy E, Charbit A: Role of the wbt locus of Francisella tularensis in lipopolysaccharide Oantigen biogenesis and pathogenicity. Infect Immun 2007, 75(1):536-541.

94. Li Q, Reeves PR: Genetic variation of dTDP-L-rhamnose pathway genes in Salmonella enterica. Microbiology 2000, 146(Pt 9):2291-2307.

95. Lundborg M, Modhukur V, Widmalm G: Glycosyltransferase functions of E. coli O-antigens. Glycobiology 2009, 20(3):366-368.

96. Mukhopadhyay R, Rosen BP, Phung LT, Silver S: Microbial arsenic: from geocycles to genes and enzymes. FEMS Microbiol Rev 2002, 26(3):311-325.

97. Sato T, Kobayashi Y: The ars operon in the skin element of Bacillus subtilis confers resistance to arsenate and arsenite. J Bacterio/ 1998, 180(7):1655-1661.

98. Forsman M, Sandstrom G, Sjostedt A: Analysis of $16 \mathrm{~S}$ ribosomal DNA sequences of Francisella strains and utilization for determination of the phylogeny of the genus and for identification of strains by PCR. Int J Syst Bacteriol 1994, 44(1):38-46.

99. Titball RW, Johansson A, Forsman M: Will the enigma of Francisella tularensis virulence soon be solved? Trends Microbiol 2003, 11(3):118-123.
100. Keim PA, Johansson D, Wagne M: Molecular epidemiology, evolution, and ecology of Francisella. In Francisella Tularensis: Biology, Pathogenicity, Epidemiology, and Biodefense. Edited by Kwaik YA, Metzger DW, Nano F, Sjostedt A, Titball R. Malden, MA: Wiley, John \& Sons, Inc; 2007:30-66. vol. 1105.

101. Svensson $K$, Larsson $P$, Johansson D, Bystrom M, Forsman M, Johansson A Evolution of subspecies of Francisella tularensis. J Bacteriol 2005, 187(11):3903-3908.

doi:10.1186/1471-2164-13-422

Cite this article as: Siddaramappa et al:: Genetic diversity within the genus Francisella as revealed by comparative analyses of the genomes of two North American isolates from environmental sources. BMC Genomics 2012 13:422.

\section{Submit your next manuscript to BioMed Central and take full advantage of:}

- Convenient online submission

- Thorough peer review

- No space constraints or color figure charges

- Immediate publication on acceptance

- Inclusion in PubMed, CAS, Scopus and Google Scholar

- Research which is freely available for redistribution 